\title{
A Neural Basis for Auditory Feedback Control of Vocal Pitch
}

\author{
Michael Smotherman, ${ }^{1}$ Shuyi Zhang, ${ }^{3}$ and Walter Metzner ${ }^{1,2}$ \\ ${ }^{1}$ Department of Physiological Science, University of California, Los Angeles, Los Angeles, California 90095-1606, ${ }^{2 B r a i n}$ Research Institute, University \\ of California, Los Angeles, Los Angeles, California 90095-1761, and ' Institute of Zoology, Chinese Academy of Sciences, Beijing 100080, People's \\ Republic of China
}

Hearing one's own voice is essential for the production of correct vocalization patterns in many birds and mammals, including humans. Bats, for instance, adjust temporal, spectral, and intensity parameters of their echolocation calls by precisely monitoring the characteristics of the returning echo signals. However, neuronal substrates and mechanisms for auditory feedback control of vocalizations are still mostly unknown in any vertebrate. We used echolocating horseshoe bats to investigate the role of the midbrain and hindbrain tegmentum for the control of call frequencies in response to changing auditory feedback. These bats accurately control the frequency of their echolocation calls through auditory feedback both when the bat is at rest [resting frequency (RF)] and when it is flying and compensating for changes in echo frequency caused by flight-induced Doppler shifts [Doppler shift compensation (DSC)]. We iontophoretically injected various GABAergic and glutamatergic transmitter agonists and antagonists into the brainstem tegmentum. We found that within the parabrachial nuclei and the immediately adjacent tegmentum, excitatory effects caused by application of the glutamate agonist AMPA or the $\mathrm{GABA}_{\mathrm{A}}$ antagonist bicuculline raised RF and the frequency of calls emitted during DSC. Bicuculline application routinely blocked DSC altogether. Alternately, inhibitory effects caused by application of either the $\mathrm{GABA}_{\mathrm{A}}$ agonist muscimol or the AMPA antagonist CNQX lowered call frequencies emitted at rest and during DSC. Such an audio-vocal feedback mechanism might share basic aspects with audio-vocal feedback controlling the pitch of vocalizations in other mammals, including the involuntary response to "pitch-shifted feedback" in humans.

Key words: audio-vocal feedback control; echolocation; horseshoe bats; Rhinolophus; Doppler shift compensation; parabrachial nuclei

\section{Introduction}

Among adult mammals, only humans, bats, and possibly cetaceans appear to require auditory feedback to maintain basic parameters of their species-specific vocalizations (Movchan, 1980; Movchan and Burikova, 1982; Rübsamen and Schäfer, 1990; Esser, 1994; Janik and Slater, 1997; McCowan and Reiss, 1997; Boughman, 1998). The neural circuitry underlying the control of mammalian vocalization is complex, and our understanding of it remains fragmentary (Jürgens, 1998, 2002). However, even less is known about how auditory feedback affects vocal motor patterns or what brain areas might be involved in audio-vocal integration (Movchan, 1984; Behrend and Schuller, 2000). Previous results suggested that a certain midbrain area, the paralemniscal tegmentum, may be involved in audio-vocal feedback control (Metzner, 1989, 1993, 1996), yet a recent lesioning study raised questions about the nature of its contributions (Pillat and Schuller, 1998). Here we present results that clearly indicate a role for a neighboring brainstem structure, the parabrachial nucleus, in the control of call frequency by horseshoe bats.

Horseshoe bats specialize in adjusting the frequency of their

\footnotetext{
Received Sept. 25, 2002; revised Nov. 20, 2002; accepted Dec. 2, 2002.

This work was supported by National Institutes of Health Grants DC02538 (W.M.) and DC00397 (M.S.), a grant from The Whitaker Foundation (W.M.), National Natural Science Foundation of China Grant 30025007 (S.Z.), and a visiting scholarship from the Chinese Academy of Sciences (W.M.). We thank Dr. M. Konishi, Dr. P. Narins, and Dr. R. Krahe for discussion and comments, Dr. K. Beeman for designing and tailoring most of the software and hardware used to simulate Doppler shifts, and Dr. Y. T. Yan for technical assistance. We are particularly grateful to the Chinese Forestry Department for issuing the export permits.

Correspondence should be addressed to Walter Metzner, Department of Physiological Science, University of California, Los Angeles, Box 951606, Los Angeles, CA 90095-1606. E-mail: metzner@ucla.edu.

Copyright $\odot 2003$ Society for Neuroscience $\quad 0270-6474 / 03 / 231464-14 \$ 15.00 / 0$
}

calls depending on the pitch of the echo signal. Their echolocation calls are characterized by the presence of a long constantfrequency component (Schnitzler, 1968; Neuweiler et al., 1987; Konstantinov et al., 1988). The frequency of calls emitted while the bat is perched is called the resting frequency (RF; Schnitzler, 1968). In an individual bat, RF is accurately maintained by closely monitoring the acoustic parameters of each returning echo (Schnitzler, 1968; Rübsamen and Schäfer, 1990). During flight, echo frequencies are shifted because of Doppler effects. The bats compensate for these shifts by either raising or lowering the frequency of subsequent calls (Schnitzler, 1968; Schuller et al., 1974; Simmons, 1974; Metzner et al., 2002; Smotherman and Metzner, 2003). This Doppler shift compensation (DSC) behavior (Schnitzler, 1968) ensures that the echo of interest remains within a narrow frequency range stimulating a region of the cochlea that is innervated by a disproportionately large neuronal population with exceptionally sharp frequency tuning, termed the "auditory fovea" (Schuller and Pollak, 1979). DSC may represent one of the most precise forms of sensorimotor integration known (Grinnell, 1989) and has been compared with visual fixation, in which eye movements keep an image of interest centered on the fovea (Schuller and Pollak, 1979). DSC can even be elicited in stationary horseshoe bats by presenting echo mimics, i.e., electronically delayed and frequency-shifted playbacks of the bat's own calls (Schuller et al., 1974).

The present study was designed to test the role of specific regions in the brainstem tegmentum suspected to be involved in the control of call frequencies (Movchan, 1980; Kirzinger and Jürgens, 1985, 1991; Metzner, 1989, 1993, 1996; Schuller and Radtke-Schuller, 1990; Holstege et al., 1997; Schuller et al., 1997; 
Pillat and Schuller, 1998). We focused on the area ventral to the inferior colliculus and medial to the nuclei of the lateral lemniscus, which included the paralemniscal tegmentum at its anterior end and the parabrachial nuclei (PB) at its posterior end. For this purpose, we systematically mapped this region using a stereotaxic approach (modified after Schuller et al., 1986) and iontophoretically injected various GABA and L-glutamate agonists and antagonists, respectively, while monitoring the effects on both RF and DSC behavior. Although we did not find any specific effects in the paralemniscal tegmentum, we found that we could dramatically alter RF and block DSC within the lateral portions of $\mathrm{PB}$. We found that both $\mathrm{GABA}_{\mathrm{A}}$ - and AMPA-type synaptic transmissions were involved in controlling call frequencies.

\section{Materials and Methods}

Fourteen greater horseshoe bats, Rhinolophus ferrumequinum, from the People's Republic of China were used. Procedures were in accordance with National Institutes of Health guidelines for experiments involving vertebrate animals and were approved by the local Institutional Animal Care and Use Committee. All 14 bats had been screened for optimum DSC behavior and were subsequently trained to compensate for artificially frequency-shifted playbacks of their own echolocation calls (details below). These bats had been chosen for the actual experiments because they consistently vocalized spontaneously and reliably compensated for sinusoidal frequency modulations $(0.03 \mathrm{~Hz}$ modulation frequency, 3 $\mathrm{kHz}$ maximum frequency shift above RF).

Acoustic playback system and behavioral test setup. Experiments were performed in an anechoic chamber $\left(28^{\circ} \mathrm{C},>50 \%\right.$ relative humidity) where echoes reflected from the walls were below the noise level of our recording system [i.e., $<45 \mathrm{~dB}$ sound pressure level (SPL)]. The electronic setup for the generation of the frequency-shifted echo mimics followed a design described previously (Schuller et al., 1974, 1975), modified by custom-built hardware and software devices (Metzner et al., 2002). Briefly, calls were recorded by a 0.25 inch ultrasonic microphone and amplifier (Brüel \& Kjær, Nærum, Denmark) positioned $15 \mathrm{~cm}$ in front of the bat's nostrils, electronically delayed by $4 \mathrm{msec}$ (custom-built delay line), heterodyned (model DS335 function generators, accuracy, $>0.01 \mathrm{~Hz}$ at $80 \mathrm{kHz}$; Stanford Research Systems, Sunnyvale, CA), highand subsequently low-passed $(99 \mathrm{kHz}$ each; digital two-channel filter, model SR650; rolloff, $115 \mathrm{~dB}$ /octave; Stanford Research Systems), and then played back via a power amplifier (model 7500; Krohn-Hite, Avon, $\mathrm{MA}$ ) and an ultrasonic condenser-type loudspeaker (Panasonic Inc., Secaucus, NJ). The transfer function of the loudspeaker allowed delivery of pure tone pulses of up to $122 \mathrm{~dB}$ SPL measured at the position of the bats' pinnae and $\pm 5 \mathrm{kHz}$ around the bats' $\mathrm{RFs}$, which ranged from 76.2 to 80.1 $\mathrm{kHz}$. Within this frequency range of $71-85 \mathrm{kHz}$, the playback system (including loudspeaker) had a frequency response of $\pm 3 \mathrm{~dB}$ and harmonic distortion for pure tone signals of $<60 \mathrm{~dB}$ SPL. Calibration of the playback system was performed with a 0.25 inch ultrasonic microphone and power amplifier (Brüel \& Kjær) using commercial signal analysis software (Signal; Engineering Design, Belmont, MA). The frequency and amplitude of the bats' calls were extracted from a custom-built frequency-to-voltage and AC-DC converter, respectively. The accuracy for determining call frequency and amplitude was $\pm 24 \mathrm{~Hz}$ and $\pm 3 \mathrm{~dB}$, respectively. Call frequency, call amplitude, and time course and amount of induced frequency shift in the echo mimic were continuously monitored and recorded on videotape using a recording adapter (3000A; Vetter, Rebersburg, PA; sample rate, $40 \mathrm{kHz} / \mathrm{channel}$ ). Subsequent analysis was performed with Signal and commercially available statistics software (SigmaStat and SigmaPlot, Jandel Scientific, San Rafael, CA). Statistical comparisons between subsets of data were performed using either Student's $t$ test or more commonly the Kruskal-Wallis one-way ANOVA on ranks.

The behavioral parameters measured were the animal's RF and its DSC response to echo mimics that were sinusoidally frequency-shifted above the bat's RF. If the playback frequency is slowly increased and then decreased in a sinusoidal manner, horseshoe bats will slowly lower and then raise their call frequency in a similarly sinusoidal manner. The rate at which playback frequency is raised and lowered is defined as the modulation frequency (Schuller et al., 1975). The maximum frequency shift presented was usually 2 or $3 \mathrm{kHz}$, and the modulation frequency was typically $0.1 \mathrm{~Hz}$, although some bats performed better at different modulation frequencies. To facilitate comparisons between bats (or the same bat) tested at different modulation frequencies and for summarizing a bat's performance over many cycles, the timing of the call frequency data can be converted from absolute time (see Fig. 1a) to the phase of echo frequency modulation (see Fig. $1 b$ ). Curve fits can then be applied to describe the mean of the bat's performance over several cycles (see Fig. $1 b$, solid line), and the accompanying $\mathrm{SE}$ of the estimate provides an indication of how well the bat followed the stimulus protocol. Because DSC is known to be highly asymmetrical, being strongly biased toward responding to echo frequencies shifted above RF, stimulus protocols were designed to be similarly asymmetrical. Intensity levels of the artificial echoes were attenuated by $10-30 \mathrm{~dB}$ relative to the intensity of the bat's calls. In addition, we also visually inspected the bats for any effects on movements of the ears or nose leaf.

In 3 of the 14 animals, we also tested the effects of the drugs on compensation behavior to natural Doppler-shifted echoes in bats that were swung on a pendulum against a large echo target (Gaioni et al., 1990). For this purpose, the bats are placed in a body mold made from soft foam and attached to the base of the pendulum immediately after the end of drug injections and the injection pipettes had been retracted from the brain. Because the effects of GABAergic drugs (muscimol and bicuculline) lasted for at least $15 \mathrm{~min}$, we could test $>30$ swings for each drug. The shorter-lasting effects of glutamatergic drugs (AMPA and CNQX), however, allowed only testing up to 10 swings before DSC and RF recovered to preinjection levels. The pendulum was suspended from the ceiling and had a length of $2.0 \mathrm{~m}$. It swung through an $\operatorname{arc}$ of $80^{\circ}(\sim 2.6 \mathrm{~m})$. The minimum distance between bat and floor was $20 \mathrm{~cm}$, which was reached at the midpoint of the swing. A large plywood target $(225 \times 125 \mathrm{~cm})$ was placed $10-15 \mathrm{~cm}$ beyond the most forward point of the swing of the pendulum. The ceiling as well as either side of the path along which the pendulum swings was lined with sound-absorbing material to reduce echoes returning from the sides. The bat's calls were monitored by a 0.25 inch ultrasonic microphone (Brüel \& Kjær) that was attached to the pendulum $7 \mathrm{~cm}$ above the bat's head and pointed toward it so that the reflection of echoes from the microphone were minimized. The calls were amplified and analyzed as describe above.

Initial training sessions of the bats consisted of $30 \mathrm{~min}$ sessions repeated daily for up to 1 week, during which the animals were accustomed to the playback setup, including being restrained in the foam holder. We presented food rewards (mealworms) for consistent DSC behavior. At the beginning and end of each session, bats were exposed to real Dopplershifted echoes by swinging them for $\sim 2$ min each on the pendulum.

During the actual experiments, acquisition of vocalization data included their frequency, duration, intensity, and repetition rate. Surgery and stereotaxic approaches followed those outlined previously (Schuller et al., 1986; Metzner, 1993, 1996). The bats were allowed to recover from surgery for $1-3 \mathrm{~d}$ before beginning the experiment, during which the bats were fully awake to vocalize spontaneously.

Iontophoresis and pharmacological agents tested. Iontophoresing electrodes were triple-barrel glass micropipettes with tips broken to inner tip diameters of $<10 \mu \mathrm{m}$. Electrodes were variously filled with different transmitter agonists and antagonists, respectively. This allowed us to affect the same cell cluster with both agonists and antagonists during the same penetration. Actions caused by the different drugs injected from one multibarrel pipette into the same brain area served as a control for one another. GABAergic drugs tested were GABA, 3-hydroxy5-aminomethylisoxazole (muscimol hydrobromide), and bicuculline methiodide. Glutamatergic drugs tested were L-glutamate, AMPA, 6-cyano-7-nitroquinoxaline-2,3-dione (CNQX), NMDA, ( \pm )-3(2-carboxypiperazin-4-yl)propanephosphonic acid (CPP), and DL-2amino-5-phosphonovaleric acid (AP-5). All drugs were obtained from Sigma (St. Louis, MO). Concentrations for all agonists and antagonists were $10 \mathrm{~mm}$ (natural transmitters, $100 \mathrm{~mm}$ ) in $165 \mathrm{~mm} \mathrm{NaCl}$. For GABAergic drugs, pH 3.2 and 100-400 nA positive current for injection 
and negative to prevent leakage; for glutamatergic drugs, $\mathrm{pH} 8.3$, negative current for injection, positive to prevent leakage. Despite these rather large amounts of current used for injections, we found that effects were limited to less than $\pm 200 \mu \mathrm{m}$ of the injection site (see Mapping of bicuculline effects).

The pipettes were placed stereotaxically (see above) before drug injections. They remained in place for the duration of the iontophoresis and also for the recovery period (while applying backing current) when we intended to inject the same drug again or to inject a different drug from one of the two other barrels of the same triple-barrel pipette at the identical site as a control. Pipettes did not remain in the brain but were retracted at the end of the daily experimental sessions and also when we tested the bat's DSC performance for real Doppler-shifted echoes on the pendulum immediately after drug injection while the drug was still effective (see above).

To unequivocally verify the site of our drug injections, in 10 of the 14 bats used here, we either made a very small permanent electric lesion with an electrode at the conclusion of the experiment (Metzner, 1993) or injected biotinylated muscimol and visualized the label histochemically (see Fig. 2; for details on biotinylation procedure, see below). Comparison of the actual location of a lesion or injection site with the stereotaxically predicted position revealed an accuracy of approximately \pm 100 $\mu \mathrm{m}$. Other injection sites could be reconstructed with reference to the histologically verified site using the stereotaxic coordinates of the corresponding penetrations.

Histochemical visualization of muscimol. Before biotinylation of muscimol (MUS), a positive charge was added by incubating MUS with bromoacetic acid. Subsequent biotinylation with biotin (long-arm) hydrazide (Vector Laboratories, Burlingame, CA) enables iontophoresis of the biotinylation product. By comparing the action of biotinylated MUS with that of unbiotinylated MUS at the same injection sites, we found that the physiological activity of biotinylated MUS did not differ from that of its unbiotinylated form. Individual neurons presumably affected by MUS were labeled, probably because of receptor internalization. Labeling was similar to that obtained with biotinylated ibotenic acid or neurobiotin and biocytin.

In the first step of the biotinylation procedure, a positive charge was added to MUS, allowing us to inject it using iontophoresis. Three solutions (A-C) were prepared. For solution A, to a $10 \mathrm{~mm}$ equivalent of MUS hydrobromide (Research Biochemicals, Natick, MA), bromoacetic acid (Sigma) was added at a 1:1 molar ratio. Substances were dissolved in acetonitrile (Sigma) for anhydrous conditions and incubated for $\sim 30$ min at $4^{\circ} \mathrm{C}$ protected from light. For solution B, biotin (long-arm) hydrazide (Vector Laboratories) was dissolved in dimethylsulfoxide to a concentration of $50 \mathrm{mg} / \mathrm{ml}$. For solution C, 1-(3-dimethylaminopropyl)3-ethyl carbodiimide- $\mathrm{HCl}$ was dissolved in $165 \mathrm{~mm} \mathrm{NaCl}$ to a concentration of $100 \mathrm{mg} / \mathrm{ml}$. Then we added $40 \mu \mathrm{l}$ of solution B to $100 \mu \mathrm{l}$ of solution $\mathrm{C} / 1 \mathrm{ml}$ of solution $\mathrm{A}$. We incubated at $4^{\circ} \mathrm{C}$ protected from light for 3-4 hr $(<20 \mathrm{hr})$. Note that the maximum concentration of MUS was limited to $\sim 20 \mathrm{~mm}$. Above this concentration, the biotin (long-arm) hydrazide polymerized when added at the corresponding molar equivalent.

Biotinylated MUS was injected using positive current (custom-built injection design; +9V DC) with electrode resistances of 5-30 M $\Omega$ (glass micropipettes with tapered tips; diameter, $5-8 \mu \mathrm{m}$ ) for $1-5 \mathrm{~min}$. Negative current was used for backing.

Animals were allowed to survive between 3 and $10 \mathrm{hr}$ after the injections. They were then killed (overdose of pentobarbital, $1 \mathrm{ml} / \mathrm{kg}$ ) and perfused transcardially with saline followed by a solution of $4 \%$ paraformaldehyde in $0.1 \mathrm{~m}$ phosphate buffer, $\mathrm{pH}$ 7.4. Brains were extracted, transferred into $0.1 \mathrm{M}$ phosphate buffer $\left(0.1 \mathrm{M} \mathrm{PO}_{4}\right.$ buffer, $\mathrm{pH}$ 7. 4), and stored at $4^{\circ} \mathrm{C}$ overnight.

Brain sections were processed according to a protocol described previously (Metzner, 1996; Metzner and Juranek, 1997). The following solutions were used: $\mathrm{PBS}\left(0.02 \mathrm{M} \mathrm{PO}_{4}\right.$ buffer, $\mathrm{pH} 7.2-7.4$, and $0.9 \% \mathrm{NaCl}$ ), $0.1 \%$ Triton X-100 in PBS (PBS-T), and 0.1 M Tris buffer (TB), pH 7.2. Brains were cut on a cryostat (immersed in embedding medium; TissueTek; Miles, Elkhart, IN) at $50 \mu \mathrm{m}$ thickness and transferred into PBS. They were then "prebleached" by soaking them for $10 \mathrm{~min}$ in $0.5 \% \mathrm{H}_{2} \mathrm{O}_{2}$ in PBS to inhibit red blood cell staining in connection with the subsequent peroxidase reaction (see below).

Using a Vectastain ABC Elite kit (Vector Laboratories), an ABC solution was prepared (four drops of A plus four drops of for $10 \mathrm{ml}$ of PBS-T) and stored for $30 \mathrm{~min}$. After prebleaching, sections were washed three times for $10 \mathrm{~min}$ in PBS and incubated in the ABC solution in small covered dishes at $4^{\circ} \mathrm{C}$ between 4 and $24 \mathrm{hr}$. Sections were then washed three times for $10 \mathrm{~min}$ in PBS and once for $10 \mathrm{~min}$ in cold $\mathrm{TB}\left(4^{\circ} \mathrm{C}\right)$ and then processed by the $3,3^{\prime}$-diaminobenzidine tetrahydrochloride (DAB) procedure. They were presoaked in a solution of $50 \mathrm{ml}$ of cold $\mathrm{TB}$, to which $0.4 \mathrm{ml}$ of an $8 \%$ nickel ammonium sulfate solution (final concentration, $0.064 \%$ ) and $50 \mathrm{mg}$ of DAB (final concentration, $0.1 \%$; Sigma) had been added. After $15 \mathrm{~min}, 30 \mu \mathrm{l}$ of $3 \% \mathrm{H}_{2} \mathrm{O}_{2}$ was added to this solution (final concentration, $0.0018 \%$ ), and the sections were incubated for 10-15 min, depending on the strength of the background label. Sections were then washed at least three times for $10 \mathrm{~min}$ in cold TB, mounted on subbed slides, counterstained with neutral red, and analyzed light microscopically. The nomenclature of brain structures followed that described for the rat brain (Swanson, 1992; Paxinos and Watson, 1998).

\section{Results}

As a reference for any effects that drug injections could have on DSC behavior, an example of a normal DSC response to frequency-shifted playback signals is given in Figure 1a. On-line recordings of the bat's calls (Fig. $1 a, V O C$ ) were sinusoidally shifted in frequency above RF (Fig. 1a, $D f$ ) before being played back after a short delay ( $4 \mathrm{msec}$ ) mimicking Doppler-shifted echoes. These echo mimics were therefore constantly changed in frequency (open-loop condition). Continuous tracking of these frequency shifts caused the bat to adjust its call frequencies accordingly and thus minimize the frequency shifts in the playback signal. When the echo mimics were delivered at frequencies above RF, the bat lowered its call frequency, and when the playback frequency fell below RF, the bat increased its call frequency toward RF (Fig. 1 $a, b$, bottom trace, filled circles). This DSC behavior caused the frequency of the echo mimics to remain within a narrow range of only a few hundred hertz around RF.

When injecting various GABA and glutamate agonists and antagonists into the brainstem tegmentum ventral to the inferior colliculus, we found that these drugs affected RF and DSC only within the lateral portions of $\mathrm{PB}$ and the immediately adjacent tegmentum. As described below in detail, depending on the drugs injected, we could raise or lower RF and reduce or even completely block DSC. We repeatedly injected these agents into PB in a total of 14 bats (up to 45 injections in an individual bat over a period of up to 6 weeks) and all experiments yielded consistent results. In the following, we present average data from those four bats in which we could test the effects of both GABAergic and glutamatergic drugs on RF and DSC in the same bats and at the same locations (see Figs. $3 a, d, 5 a, d$, box plots, Tables 1, 2). In addition, we show representative examples of averaged traces of DSC performance from one individual bat (RF28) (see Figs. 3b,c, 5b,c).

In 10 of the 14 animals used in our study, we were able to histologically verify the area where iontophoresis of various transmitter agonists and antagonists had the strongest effects on $\mathrm{RF}$ and DSC by either injecting biotinylated muscimol or setting small electrolytic lesions at the termination of the experiments. All sites were located within the lateral PB (Fig. $2 a$; total of five cases) or at $<200 \mu \mathrm{m}$ distance within the midbrain tegmentum immediately adjacent to PB (Fig. $2 b$; total of five cases) as defined in Nissl-stained material. From systematically mapping the area (for details, see below, Figs. 7, 8), we estimate the area in which we could affect RF and DSC behavior to cover $\sim 700 \mu \mathrm{m}$ rostrocaudally, $600 \mu \mathrm{m}$ dorsoventrally, and $300 \mu \mathrm{m}$ mediolaterally. In the 

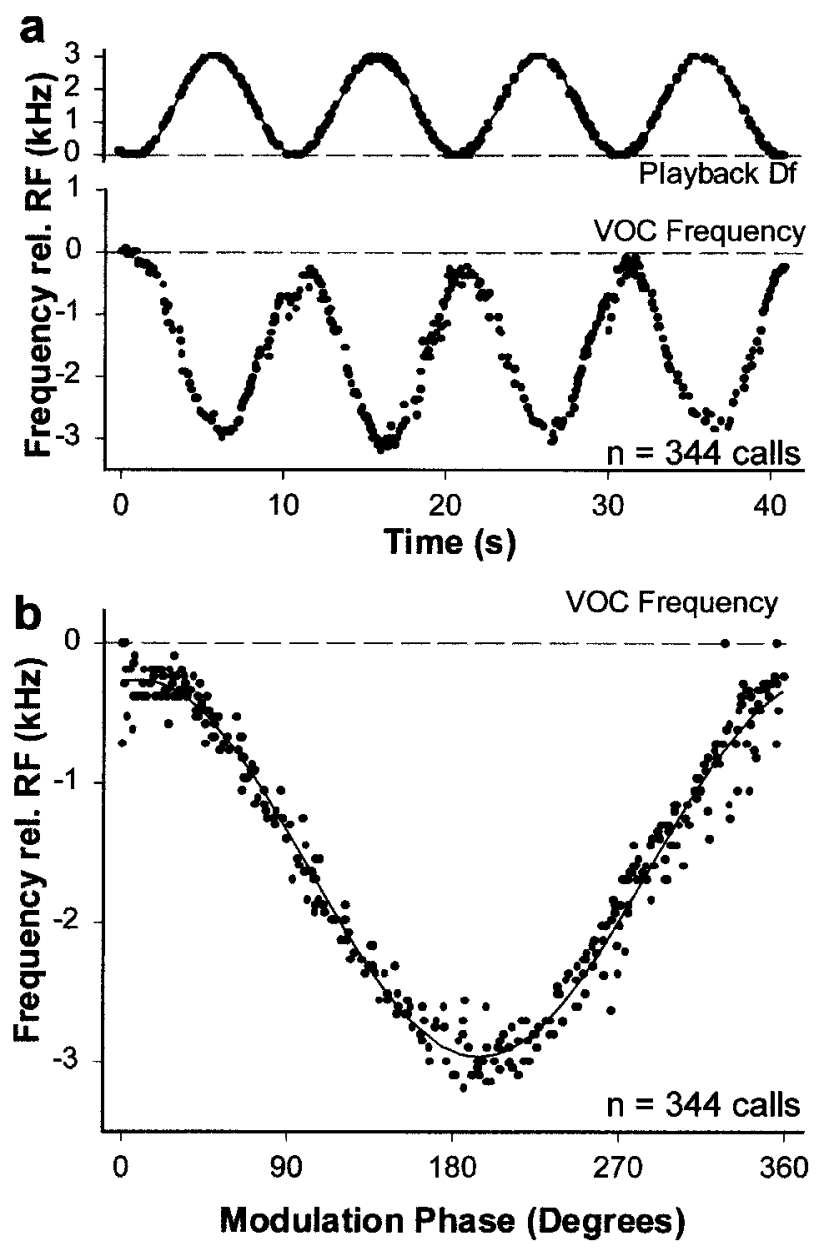

Figure 1. DSC response to artificially frequency-shifted echo mimics. $a$, The bat's calls (VOCFrequency, bottom trace) were recorded in real time, electronically sinusoidally modulated in frequency (Playback Df, top trace) above the bat's RF (dashed horizontal lines), and played back to the bat with a 4 msec delay. As a result, the bat sinusoidally modulated its call frequency below RF. In the bottom trace, each symbol represents the maximum frequency detected within one call. Symbols in the top trace indicate the corresponding value of the frequency shift presented at that time. All frequencies are given relative to the bat's median RF (77.41 kHz). Maximum frequency shift of playbacks, $3.2 \mathrm{kHz}$; modulation frequency, $0.1 \mathrm{~Hz}$. Total number of calls analyzed, $n=344$. b, Same data as in $a$, bottom trace, but plotted relative to the phase of each of the four sinusoidal frequency modulations of the Playback Df ( $a$, top trace). The black curve represents the nonlinear curve fit through the data.

following, we will refer to the lateral portions of the parabrachial nuclei and the immediately adjacent tegmentum as PB.

\section{Effects of GABAergic drugs}

Typical effects of the $\mathrm{GABA}_{\mathrm{A}}$ agonist MUS and antagonist bicuculline methiodide (BMI) on the bat's RF and DSC behavior are shown in Figure 3 and Table 1. Unilateral injection of MUS into PB caused RF to drop significantly (Fig. $3 a$ ). Although the preinjection RF was maintained at a fairly stable value, usually varying less than $\pm 100 \mathrm{~Hz}$ around its median, values for RF after MUS injection became less stable and showed a fivefold increase in SD, varying over a total range of $>3 \mathrm{kHz}$ (Fig. $3 a$, Table $1 a$ ). Despite this increased range of call frequencies emitted at rest, virtually no calls were produced above or even at the original, preinjection RF (Fig. 3a). Bilateral MUS injections caused RF to decrease even further on average by $>1 \mathrm{kHz}$ below the preinjection value (data not shown). Conversely, unilateral iontophoresis of BMI signifi- cantly increased RF above the preinjection value. RF also became less stable, varying by up to $2.12 \mathrm{kHz}$ (Fig. $3 a$, Table 1). Nevertheless, very few calls ( 28 of a total of 1335 calls) were produced at frequencies at or below the preinjection RF. Typically, the effects of MUS or BMI began 10-30 sec after injection onset. All effects were reversible within $2 \mathrm{hr}$ for MUS and $20 \mathrm{~min}$ for BMI after the iontophoresis current had been turned off. On several occasions $(>10)$, we repeated injections of BMI while leaving the injection pipette in place and the current reversed to prevent leakage of the drug during the recovery period. (We chose BMI over MUS because of the faster recovery to BMI injections.) The subsequent injections always yielded results that were virtually identical, and three such subsequent BMI injections are incorporated in the data set used in Figure $3 a$.

During DSC behavior elicited by the same frequency shifts in the played back echo mimics as in the control condition, injection of MUS caused the bat to lower its call frequencies by an amount greater than before MUS injection; i.e., the "compensation depth" increased (Fig. 3b,d, Table 1). The animals even "overcompensated"; i.e., they lowered their call frequency by an amount that was on average $34 \%$ greater than the frequency shift presented in the playback signal (Table 1). MUS thus increased the overall range of call frequencies emitted almost 1.7-fold (3.25 vs $1.93 \mathrm{kHz}$ ) (Table 1). DSC behavior became overall also more erratic (Fig. 3b). Iontophoresis of the natural transmitter GABA had effects that were virtually identical to those of MUS $(n=3$ bats, 10 injections). BMI injections, on the other hand, prevented the bat from lowering its call frequencies below RF; hence DSC behavior appeared to be virtually eliminated (Fig. $3 c, d$ ). The mean call frequency emitted after BMI injections rose almost 200 $\mathrm{Hz}$ above RF (Table 1).

To examine whether the bats failed to show DSC behavior after drug injections merely because these agents affected the motivational state of the animal, thus masking DSC, we also tested the compensation behavior after drug injection while the bat was swinging on a pendulum against a large background target (Gaioni et al., 1990). (Note that the injection pipettes had been retracted, and the animal had been removed from the experimental setup used to test for artificially frequency-shifted echo mimics; for details, see Materials and Methods.) The exposure to naturally Doppler-shifted echoes normally elicited consistent calling behavior and DSC in even the least cooperative bats. We found in all three animals tested in this manner that MUS and BMI injections into $\mathrm{PB}$ and subsequent exposure to real Doppler-shifted echoes during pendulum swings resulted in changes in call frequency that were virtually identical to those seen after drug injections in the same bats responding to artificially frequency-shifted playback signals in the experimental setup (Fig. 3); MUS lowered call frequencies well below normal levels and caused an increase in the compensation depth, whereas BMI increased call frequencies above RF, virtually abolishing DSC.

A detailed spectral analysis of the bats' calls showed that, much like during natural DSC, the typical spectral composition of the bats' calls remained unchanged; i.e., the entire calls were frequency-shifted, and frequency compensation did not occur during emission of an individual call but instead only from call to call (Schnitzler, 1968). This is illustrated in Figure 4. A typical horseshoe bat call is depicted in Figure $4 a$. It usually begins with a brief upward frequency modulation, followed by the characteristic long constant-frequency component, and terminates with a short downward frequency-modulated portion (Schnitzler, 1968; Neuweiler et al., 1987). Injection of MUS (Fig. 4b) or BMI 
Table 1. Average effects of MUS and BMI on RF and DSC

\begin{tabular}{|c|c|c|c|c|c|c|c|}
\hline Group & $\begin{array}{l}\mathrm{RF}(\mathrm{Hz}) \\
(\text { mean } \pm \mathrm{SD})\end{array}$ & $\begin{array}{l}\text { RF median } \\
(\mathrm{Hz})\end{array}$ & $\begin{array}{l}\text { Maximum } \\
\text { frequency }(\mathrm{Hz})\end{array}$ & $\begin{array}{l}\text { Minimum } \\
\text { frequency }(\mathrm{Hz})\end{array}$ & $\begin{array}{l}\text { Overall } \\
\text { range } \\
(\mathrm{Hz})\end{array}$ & $\begin{array}{l}\text { Maximum } \\
\text { compensation } \\
\text { depth (\%) }\end{array}$ & $n$ \\
\hline \multicolumn{8}{|l|}{ RF } \\
\hline Control & $0 \pm 82$ & 43 & 212 & -548 & 760 & & 1004 \\
\hline MUS & $-502 \pm 410$ & -42 & 375 & -2704 & 3079 & & 963 \\
\hline BMI & $316 \pm 199$ & 27 & 936 & -1182 & 2118 & & 1335 \\
\hline \multicolumn{8}{|l|}{ DSC } \\
\hline Control & $-1237 \pm 510$ & -1314 & -205 & -2135 & 1930 & 92 & 834 \\
\hline MUS & $-1853 \pm 702$ & -1961 & -220 & -3470 & 3250 & 134 & 901 \\
\hline BMI & $174 \pm 357$ & 26 & 1037 & -567 & 1604 & 2 & 1131 \\
\hline
\end{tabular}

$\mathrm{RF}$ is same data set as in Figure $3 a$; DSC is same data set as in Figure $3 d$ (four bats with three injections per bat). Means and medians are relative to the average control RF (78.48 $\pm 0.089 \mathrm{kHz}$ ). Maximum and Minimum are relative to their respective medians. Maximum compensation depth is the ratio of the maximum amount of lowering of call frequency relative to the maximum frequency shift in the echo playback (100\% corresponding to complete compensation). All MUS and BMI values are significantly different from control $(p<.001)$.

Table 2. Average effects of AMPA and CNQX on RF and DSC

\begin{tabular}{|c|c|c|c|c|c|c|c|}
\hline Group & $\begin{array}{l}\mathrm{RF}(\mathrm{Hz}) \\
(\text { mean } \pm \mathrm{SD})\end{array}$ & $\begin{array}{l}\text { RF median } \\
(\mathrm{Hz})\end{array}$ & $\begin{array}{l}\text { Maximum } \\
\text { frequency }(\mathrm{Hz})\end{array}$ & $\begin{array}{l}\text { Minimum } \\
\text { frequency }(\mathrm{Hz})\end{array}$ & $\begin{array}{l}\text { Overall } \\
\text { range } \\
(\mathrm{Hz})\end{array}$ & $\begin{array}{l}\text { Maximum } \\
\text { compensation } \\
\text { depth (\%) }\end{array}$ & $n$ \\
\hline \multicolumn{8}{|l|}{ RF } \\
\hline Control & $0 \pm 70$ & 41 & 141 & -548 & 689 & & 1005 \\
\hline AMPA & $233 \pm 130$ & 273 & 246 & -1229 & 1475 & & 969 \\
\hline CNQX & $-390 \pm 440$ & -272 & 563 & -3451 & 4014 & & 919 \\
\hline \multicolumn{8}{|l|}{ DSC } \\
\hline Control & $-1192 \pm 518$ & -1231 & 178 & -2135 & 2313 & 79 & 925 \\
\hline AMPA & $-293 \pm 290$ & -271 & 483 & -1113 & 1596 & 18 & 1139 \\
\hline CNQX & $-1560 \pm 478$ & -1550 & 46 & -3030 & 3076 & 56 & 1204 \\
\hline
\end{tabular}

RF is same data set as in Figure $5 a$; DSC is same data set as in Figure $5 d$ (four bats, three injections per bat). Same convention as in Table 1. All AMPA and CNQX values are significantly different from control ( $p<.001)$.
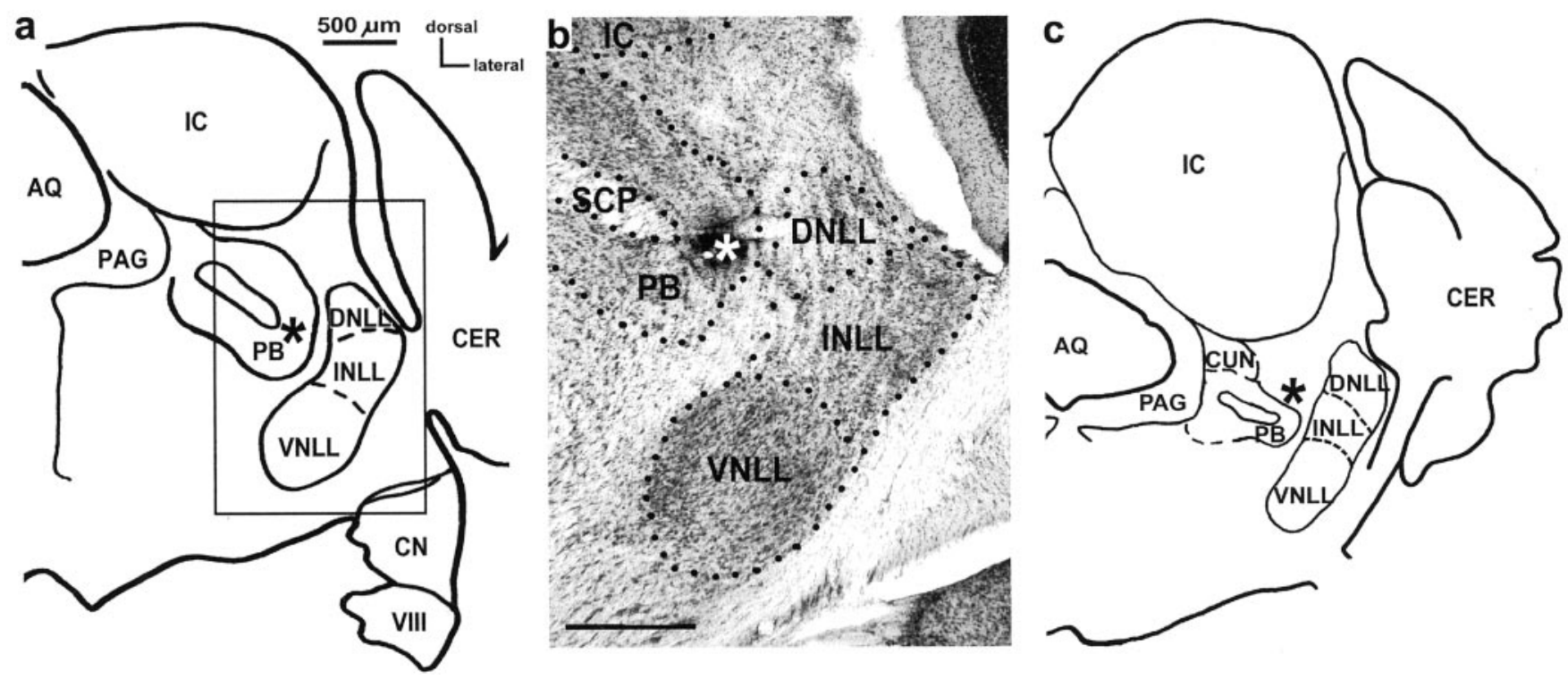

Figure 2. Schematic overviews ( $a, c)$ and histograph ( $b$; Nissl stain) of frontal sections through the brainstem showing histological verifications of a muscimol injection site localized within $\mathrm{PB}(a)$ and one at a distance of $<200 \mu \mathrm{m}$ within the immediately adjacent tegmentum (c). Biotinylated muscimol was injected for 5 min in each case (injection currents, $100 \mathrm{nA}$ ) and subsequently visualized histochemically. The injection center is denoted by an asterisk. VII, Auditory nerve; $A Q$, cerebral aqueduct; $C E R$, cerebellum; $C N$, cochlear nucleus; $C U N$, cuneiform nucleus; DNLL, dorsal nucleus of the lateral lemniscus; INLL, intermediate nucleus of the lateral lemniscus; VNLL, ventral nucleus of the lateral lemniscus; IC, inferior colliculus; $S C P$, superior cerebellar peduncle.

(Fig. 4c) into PB did not alter this pattern. The only significant change elicited by these agents was the overall shift of the call toward higher or lower frequencies causing the constantfrequency component to fall below RF (for MUS) (Fig. 4b) or above RF (for BMI) (Fig. 4c). It is worth mentioning, however, that BMI could indeed dramatically modify the spectral composition of the calls when injected into a quite different area, which is presumably homologous to the principal sensory nucleus of the trigeminus (see below; Fig. 9).

In summary, these results so far indicate that within $\mathrm{PB}$, $\mathrm{GABA}_{\mathrm{A}}$-type synaptic transmission lowered call frequencies emitted at rest and during DSC. Blockage of this type of synaptic connection by BMI eliminated the ability of the bat to lower its call frequency, thus also abolishing DSC. Such an inhibitory con- 

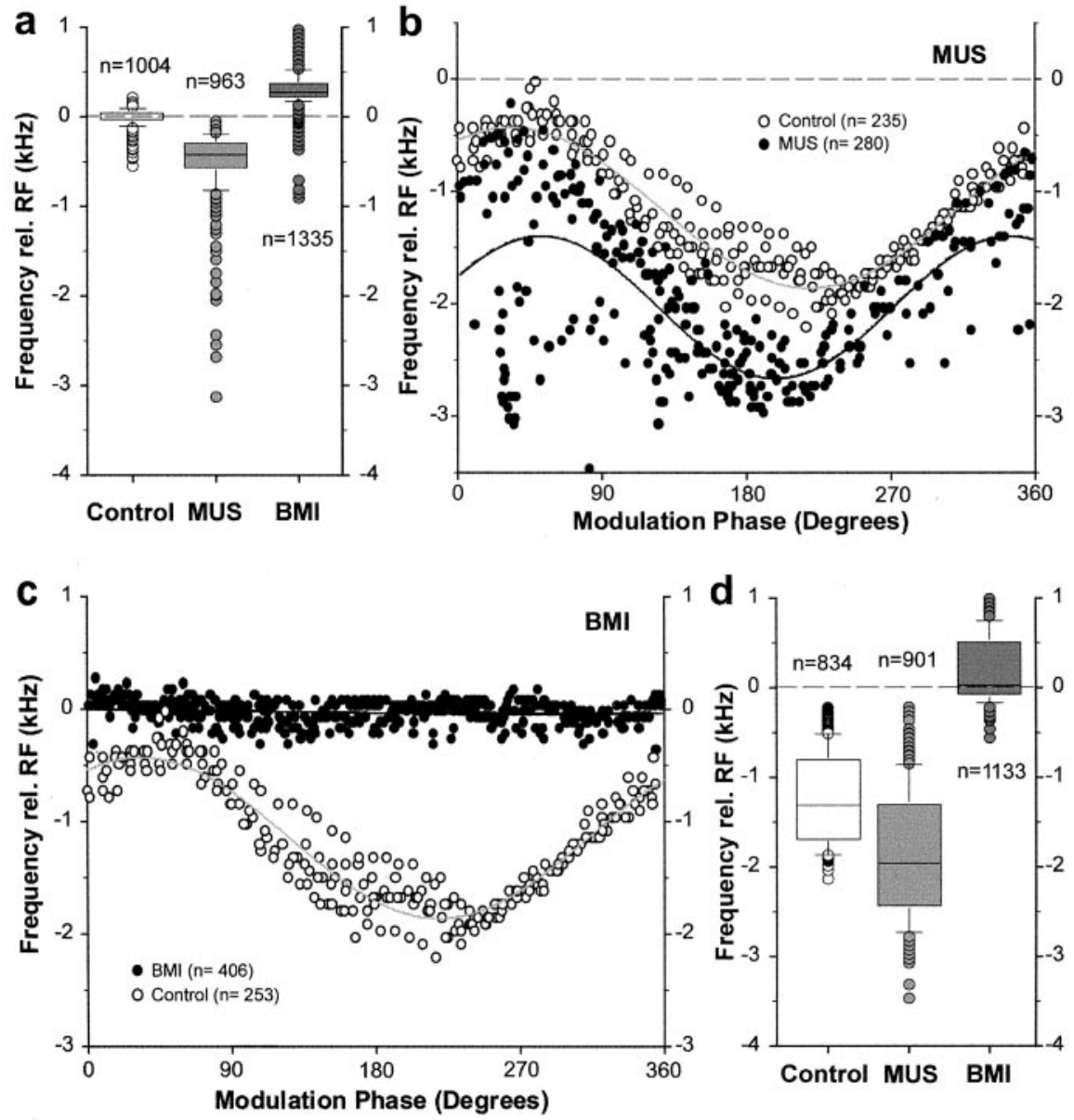

Figure 3. Effects of $\mathrm{GABA}_{\mathrm{A}}$ agonist MUS and antagonist BMl on $\operatorname{RF}(a)$ and $\mathrm{DSC}(b, c) \cdot a$, Unilateral iontophoresis of MUS and BMI and resulting changes of RF compared with control condition (i.e., RF before injections). Data represent the average of four bats; three injections of each of the drugs per bat. Bottom and top ends of boxes indicate the 25 th and 75 th percentiles, respectively, with a solid horizontal line at the median. Error bars indicate the 10th and 90th percentiles, and dots indicate the outliers. All data are normalized relative to the preinjection RF (see Table 1). The differences between all three groups were statistically significant; $p<$ 0.001. b, MUS effects on DSC ( filled circles; black line represents curve fit; SE, 0.434). A representative example from one bat is shown. Same convention as in Figure $1 b$. In addition, the bat's normal DSC immediately before MUS application is given (open circles; gray line indicates curve fit; SE, 0.183 ). Total number of calls analyzed, $n=235$ before injection and 280 for MUS injection. $\mathrm{RF}, 77.41 \pm 0.131 \mathrm{kHz}$. Maximum frequency shift in playback, $2.2 \mathrm{kHz}$. c, Effects of BMI on DSC. Same bat and site as in $b . n=253$ calls for control and 406 for BMI (5 cycles each). Preinjection curve fit ( gray curve, open circles) SE, 0.183; BMI (black curve, closed circles) SE, 0.099. Maximum frequency shift in playback, $2.2 \mathrm{kHz}$. d, Average compensation depth for four bats (same bats as in $a$; 5 compensation cycles for each bat, 3 injections per bat) plotted relative to median RF. The differences between all groups were statistically significant; $p<0.001$. Also see Table 1 .

trol mechanism had been proposed previously on the basis of physiological and anatomical evidence obtained in the paralemniscal tegmentum (Metzner, 1989, 1993, 1996). A purely inhibitory feedback mechanism controlling DSC is also consistent with results from all previous behavioral studies (Schnitzler, 1968; Schuller et al., 1974, 1975; Schuller and Pollak, 1979; Tian and Schnitzler, 1997). Recently, however, we had reassessed the effects of auditory feedback from various echo frequencies on the control of call frequencies emitted during DSC (Metzner et al., 2002). Revising our original hypothesis (Metzner, 1989, 1993), the new behavioral results suggested that inhibitory as well as excitatory feedback control call frequencies; lower call frequencies should be caused not only by an increased amount of inhibition, as seen in the pharmacological results presented in Figure 3, but also by a reduced amount of excitation (Metzner et al., 2002). This prompted us to also test the effects of glutamatergic agonists and antagonists on RF and DSC behavior at the same sites where we had previously tested MUS and BMI.

Effects of glutamatergic drugs

Indeed, we found that L-glutamate increased RF and virtually blocked any DSC response to frequency-shifted echo mimics. A more detailed analysis showed that the AMPA receptor subtype was involved; whereas NMDA and its antagonists CPP and AP-5 did not significantly alter RF or DSC ( $n=2$ bats, four experiments per bat per drug), AMPA and its antagonist CNQX did (Fig. 5, Table 2).

At the same sites where MUS and BMI had affected call frequencies, unilateral injection of AMPA resulted in a small but significant rise of RF by an average of 273 $\mathrm{Hz}$ above the preinjection value (Fig. 5, Table 2). CNQX, on the other hand, significantly lowered RF by on average $>270$ $\mathrm{Hz}$ and a maximum of $>3.4 \mathrm{kHz}$ (Fig. 5 , Table 2). Both agents also rendered the postinjection RF less stable (see larger SD and minimum and maximum values in Table 2, Effects on RF). Typical latencies for the effects of AMPA or CNQX were $<5 \mathrm{sec}$ after injection onset. All effects were reversible within $10 \mathrm{~min}$ after injections had stopped.

During DSC behavior, injection of AMPA dramatically reduced the DSC response and maintained call frequencies closer to RF than normal (Fig. 5d, Table 2 ). The overall range over which call frequencies varied during AMPA iontophoresis lessened to 0.7 times the normal range ( $1.57 \mathrm{vs} 2.31 \mathrm{kHz}$ ) (Table 2), and the maximum compensation depth shrank to less than one-fifth of its normal value (18 vs 79\%) (Table 2). Injection of CNQX, on the other hand, lowered the call frequencies during DSC by an average of $>300 \mathrm{~Hz}$ below normal levels (Fig. $5 c, d$, Table 2). In addition, the range of call frequencies increased by one-third ( 3.08 vs $2.31 \mathrm{kHz}$ ) (Table 2), and DSC behavior became less consistent (Fig. $5 c$ ).

When testing the bat's DSC on a swinging pendulum after injections of AMPA or CNQX into $\mathrm{PB}$, we found results that were consistent with those described above for responses to electronically frequency-shifted playback signals; AMPA caused call frequencies to remain closer to or even above RF and almost completely abolished DSC, whereas CNQX decreased vocalization frequencies below normal compensation levels (data not shown).

Hence, at the behavioral level, the effects of the glutamatergic agonist AMPA qualitatively resembled those seen in response to the GABAergic antagonist BMI. Conversely, the glutamatergic antagonist CNQX had effects analogous to those of the GABAergic agonist MUS; both AMPA and BMI increased RF, whereas CNQX and MUS lowered it (Figs. 3, 5). In addition, similar to MUS and BMI, AMPA and CNQX effects were fully reversible 
a
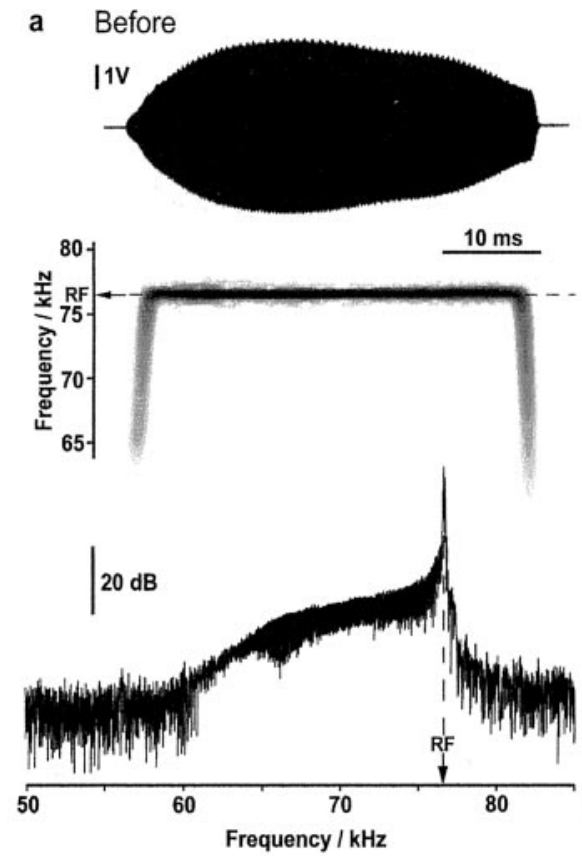

b
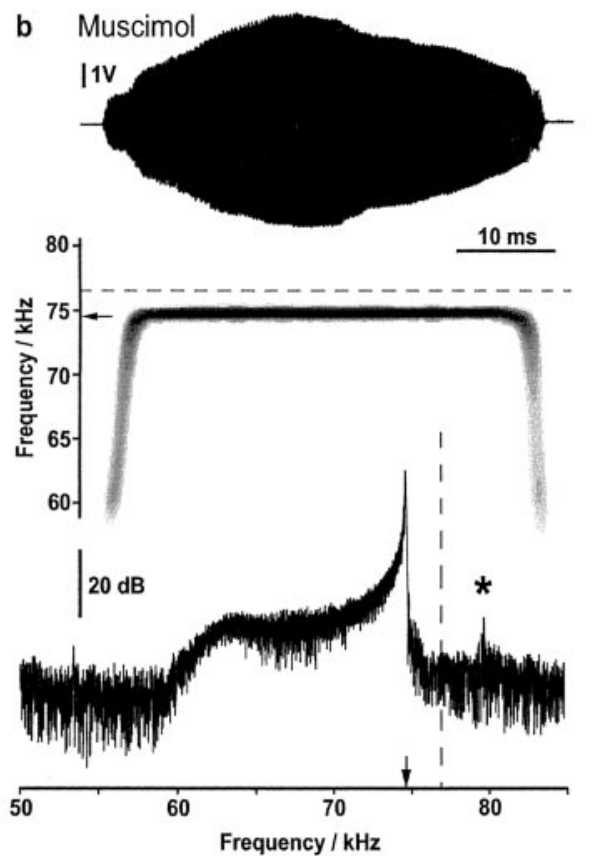

c
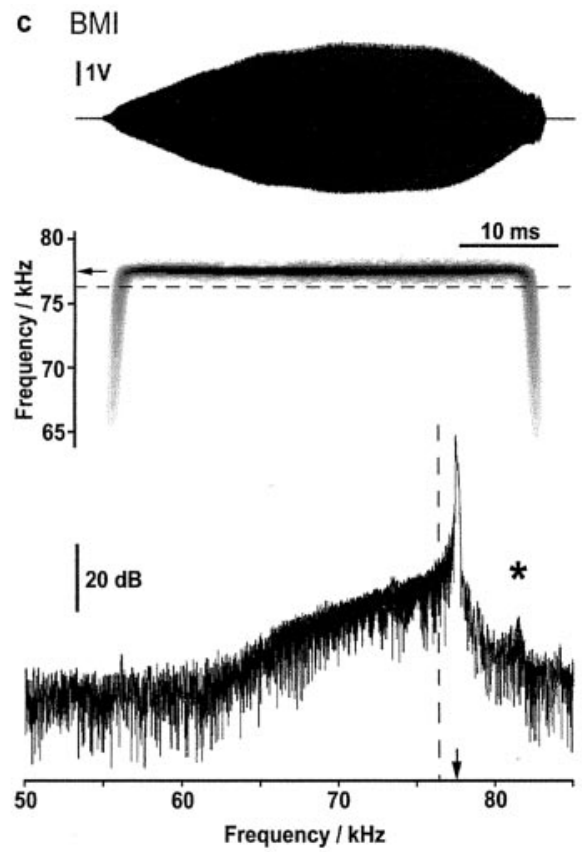

Figure 4. Effects of MUS and BMI on the spectral composition of echolocation calls. Top trace, Envelope; middle trace: sonogram (sample rate, $250 \mathrm{kHz}$; time resolution, 2 msec; frequency resolution, $244 \mathrm{~Hz}$ ); bottom trace, spectrum (sample rate, $250 \mathrm{kHz}$; frequency resolution, $7.63 \mathrm{~Hz}$ ). Asterisks in spectra of $b$ and c reflect the playback signal (which had been strongly attenuated during this recording to minimize artifacts in the spectral analysis). $a$, Typical echolocation call of greater horseshoe bats emitted at RF and recorded before drug injection. $b$, MUS shifted the entire call below preinjection frequency levels, leaving the spectral composition within the normal range of variability. c, BMl increased the entire spectrum above preinjection RF. No significant change of the spectral composition was found.

and only shifted the frequency of the constant-frequency component without altering the overall spectral composition of the echolocation calls.

Effects of simultaneous application of bicuculline and CNQX The results presented so far indicate that within $\mathrm{PB}$, the control of call frequencies is mediated by both $\mathrm{GABA}_{\mathrm{A}}$-type inhibitory and AMPA-type excitatory synaptic transmission. To further analyze this antagonistic control mechanism, we monitored how RF and DSC were affected by simultaneously applying the two antagonists, BMI and CNQX. We found that the effects of the combined antagonist application on RF as well as DSC were additive (Fig. 6): Initial injection of BMI, for example, resulted in the usual rise of RF (Fig. $6 a$; also see Fig. $3 a$ ). When CNQX was added, RF did not remain at the elevated level but, instead, returned to an intermediate level close to the original RF (Fig. 6a), also significantly increasing the variability of call frequencies. Similarly, when CNQX was injected first, and BMI was added at a later point, the initial lowering of RF caused by CNQX was abolished by additional injection of BMI, and call frequencies were emitted around the original RF, again with much larger variability than before any of the injections of only one antagonist (Fig. $6 a$ ). During DSC behavior, initial CNQX injection prevented the bat from raising its call frequencies in the characteristic manner (Fig. $6 b$; also see Fig. $5 c$ ). Additional injection of BMI abolished DSC entirely, and call frequencies were generated around the original RF, however, with a large variability (Fig. 6b,c). Conversely, initial BMI injection caused the rise in call frequency and virtually completely blocked DSC, as already depicted in Figure $3 c$. Additional injection of CNQX antagonized the BMI effect, and call frequencies were produced around the original value for RF, which was virtually identical to the outcome of the reversed injection sequence as described above (Fig. 6b,c).

\section{Mapping of bicuculline effects}

Histological verification of individual injection sites where these drugs yielded significant effects on call frequency showed that the injection centers were located within the lateral portions of the parabrachial nucleus and up to $200 \mu \mathrm{m}$ within the adjacent tegmentum (Fig. 2). We also knew that wherever, for instance, BMI was effective, its agonist MUS and also the glutamatergic agents AMPA and CNQX were as well (Fig. 6). How localized were these effective sites, however? For this purpose, we quantified the rise in $\mathrm{RF}$ caused by BMI injections into different areas of the brainstem tegmentum beneath the inferior colliculus and medial to the nuclei of the lateral lemniscus extending from the paralemniscal tegmentum at its rostral end to areas caudal to the parabrachial nuclei (Fig. $7 a, b o x$ ). We chose to analyze the changes in RF because they were always accompanied by changes in DSC (Figs. 3,5 , Tables 1,2) but required less time for data collection, thus allowing for a larger sample size. (DSC analysis involved averaging over several DSC cycles, each lasting $\geq 10 \mathrm{sec}$, whereas RF changes could be reliably quantified with call sequences of $\leq 30$ sec.) In addition, changes in RF were significantly less variable and could be quantified more easily than changes in DSC (Figs. 3, 5). Finally, we focused on the effects of BMI, because it caused RF increases, which fluctuated less than the decreases in RF caused by MUS or CNQX (Figs. $3 a, 5 a$ ) but was not toxic like AMPA, which also caused rises in RF.

We found that subsequent injections of BMI at a rostrocaudal or mediolateral distance of only $200 \mu \mathrm{m}$ could cause significantly different effects when limiting the injection time to $60 \mathrm{sec}$. Hence we used a series of 60-sec-long BMI injections to systematically map their effects within an area covering $\sim 1750 \mu \mathrm{m}$ rostrocaudally, $800 \mu \mathrm{m}$ mediolaterally, and $1100 \mu \mathrm{m}$ dorsoventrally around PB in steps of $250 \mu \mathrm{m}$. We ensured that call frequencies had returned to normal, which typically took $>20 \mathrm{~min}$, before 

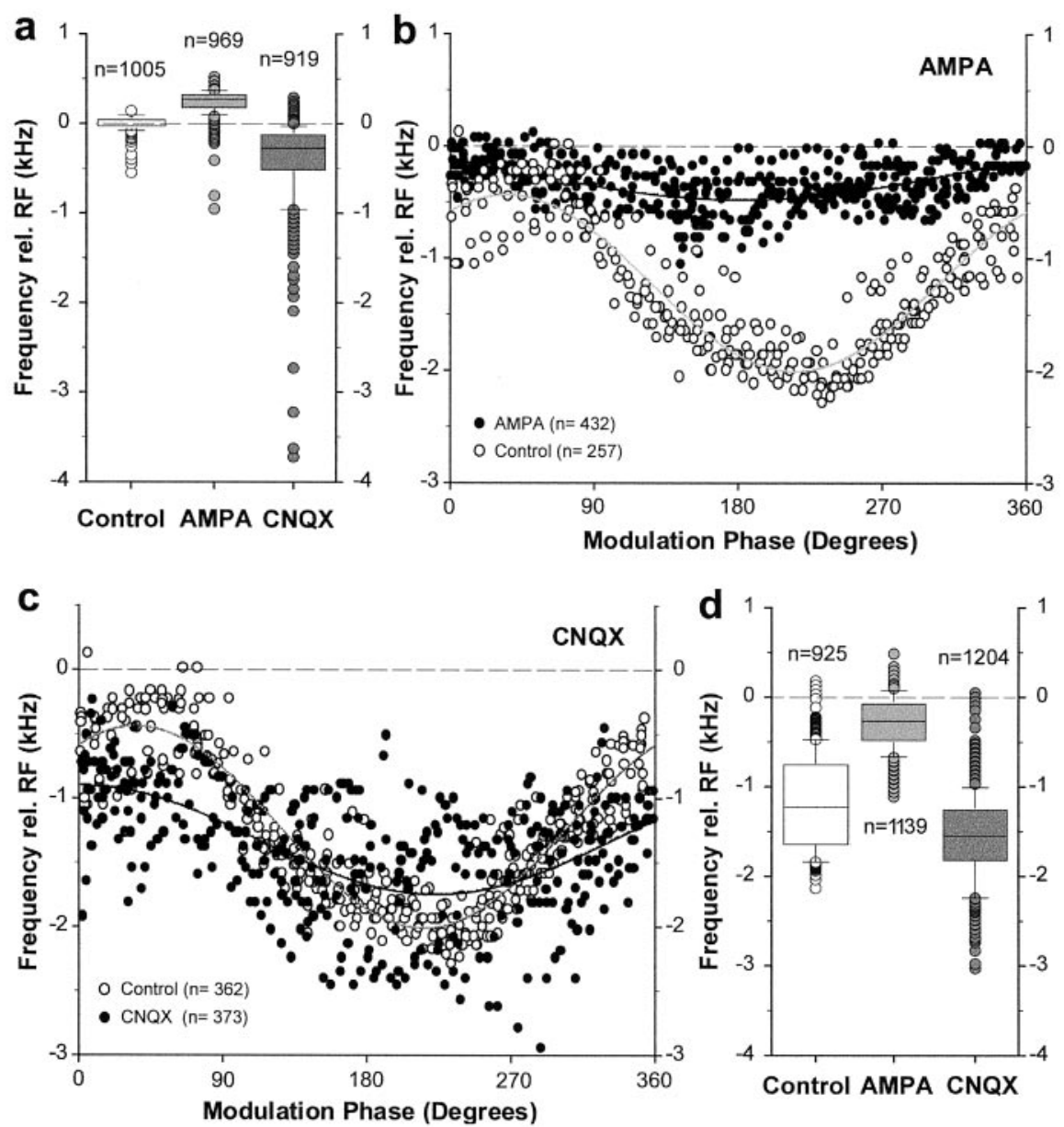

Figure 5. Effects of glutamate agonist AMPA and antagonist $\operatorname{CNQX}$ on $\operatorname{RF}(a)$ and DSC $(b-d)$. Same convention as in Figure 3. $a$, Unilateral iontophoresis of AMPA and CNQX and resulting changes of RF compared with control condition. Average of four bats, three injections per bat (same bats as in Fig. 3a, d). The differences between all three groups were statistically significant; $p<$ 0.001. b, AMPA effects on DSC ( filled circles; black line represents curve fit; SE, 0.395). Representative example from same animal and injection site as in Figure $3, b$ and $c$. In addition, the bat's normal DSC immediately before AMPA application is given (open circles; gray line indicates curve fit; SE, 0.185). $n=257$ for preinjection and 432 for AMPA injection. Median preinjection RF, $77.41 \pm 0.131 \mathrm{kHz}$; maximum frequency shift in playback, $2.2 \mathrm{kHz}$. C, Effects of CNQX on DSC. Same bat and site as in $b$ and Figure $3, b$ and $c . n=362$ calls for control and 373 for CNQX ( 5 cycles each). For curve fit to preinjection DSC ( gray curve, open circles), SE, 0.188; for CNQX (black curve, closed circles), SE, 0.545. Maximum frequency shift in playback, $2.2 \mathrm{kHz}$. d, Average compensation depth for four bats ( 3 injections per bat; same bats as in $a ; 5$ compensation cycles for each bat) plotted relative to the bats' average median RF. The differences between all groups were statistically significant; $p<0.001$. Also see Table 2 .

testing BMI effects at subsequent sites. We then reconstructed the injection sites in three dimensions based on histological verification of biotinylated MUS (Fig. 2) or electrolytic lesions as a reference using our stereotaxic approach. In two bats, we were able to survey completely the area outlined above, with similar patterns emerging. The resulting data points $(n=172$ total injections) were pooled to produce a representative threedimensional illustration (Figs. 7, 8) of the distribution of the most effective injection sites within this region of the brainstem. For eight other bats, a most sensitive injection site was also determined, although without a similarly rigorous survey of the more distantly surrounding brain areas, and the stereotaxically reconstructed injection sites for these bats are also included in Figure 8 (white stars) for comparison.

BMI injections did not yield any effects on the bat's call frequency in the anterior and central portions of the paralemniscal tegmentum (Figs. $7 a, b, 8 a_{1}, b$ ). This is consistent with findings from a previous study (Pillat and Schuller, 1998), in which nei- ther electrolytic lesions nor injections of kainic acid into these tegmental areas affected the bat's call frequency. BMI did, however, increase call frequencies when injected in more caudal tegmental regions, which correspond to the lateral portions of PB (Figs. $7 a, c, 8 a_{2}-a_{5}, b$ ) and the immediately adjacent tegmentum (Fig. 2b). Our mapping study indicated that the area affected by BMI was fairly restricted. It expanded in an anteroposterior direction from a ventromedially located area at a depth between $\sim 3200$ and $3400 \mu \mathrm{m}$ to a region that was $\sim 600 \mu \mathrm{m}$ more dorsal, $300 \mu \mathrm{m}$ more lateral, and $700 \mu \mathrm{m}$ more caudal (Fig. $8 a_{2}-a_{5}, b$ ). Because of the heterogeneous organization of this tegmental region, including the PB itself, consisting of numerous nuclei and subnuclei (Swanson, 1992; Paxinos and Watson, 1998), it was difficult to unequivocally align the pharmacologically identified areas shown in Figure 8 with distinct anatomical boundaries. When reconstructing the location of histologically verified individual injection sites, such as those given in Figure 2, we found that the areas affected by BMI mostly overlapped with dorsal and ventral aspects of the lateral portion of $\mathrm{PB}$ and the immediately adjacent tegmentum, as we had suspected previously from the histological verification of various injection sites. Because the detailed architecture of the $\mathrm{PB}$ is not known in any bat, we refrain from homologizing these areas with particular subnuclei known in PB of other mammals.

\section{Effects of bicuculline on spectral composition of calls}

Injections of GABAergic and glutamatergic agents into $\mathrm{PB}$ caused a shift in the dominant constant-frequency component of the bat's calls emitted at rest or during DSC but left the overall spectral composition of the calls unaffected (Fig. 4). This reflects call frequency changes observed during natural DSC behavior; horseshoe bats compensate for a Doppler-shifted echo signal only in the subsequent call and do not adjust their call frequency while emitting an individual call (Schnitzler, 1968).

Interestingly, however, we were able to also significantly alter the frequency composition of calls by injecting BMI into a site located $\sim 500 \mu \mathrm{m}$ caudal and $200 \mu \mathrm{m}$ ventral to the most caudal portions of PB depicted in Figures 7 and 8. Although the following results are preliminary, we believe the stark contrast to the effects that the same drug had in PB highlights the behavioral specificity of the effects observed in PB.

When we injected BMI into an area caudoventral to $\mathrm{PB}$ using the same concentration and iontophoretic settings that we had used within PB (often even with the same micropipette), we found dramatic effects on virtually all call parameters, including their envelope and temporal and spectral composition (13 injec- 

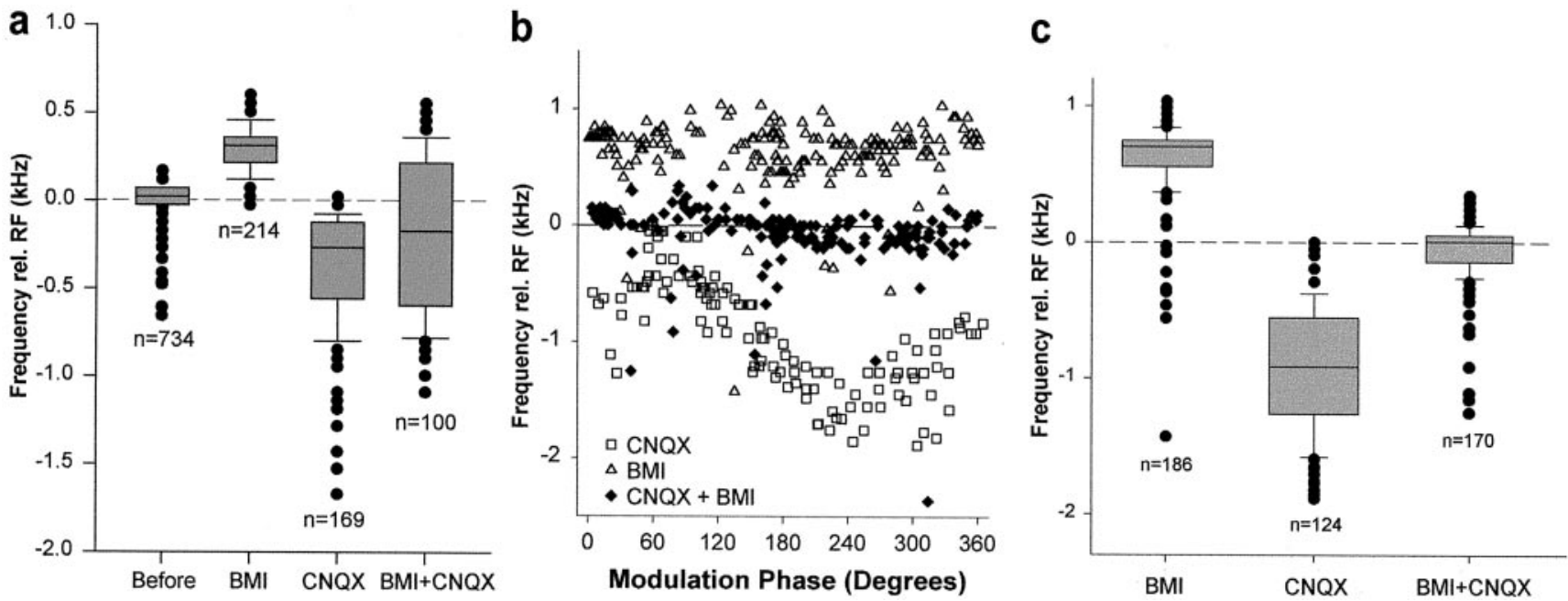

Figure 6. Combined effects of BMl and CNQX. Representative data are from one animal (same animal as in Figs. $3 b, c, 5 b, c$ ). $a$, Average effects on RF. BMI alone (median, $312 \mathrm{~Hz}$ above original RF; also see Fig. 3a), CNQX alone (median, $296 \mathrm{~Hz}$ below original RF; also see Fig. $5 a$ ), and simultaneous injection of BMI and CNQX (median, $170 \mathrm{~Hz}$ below original RF) are shown. All data were collected during the same penetration and for the same injection site. The temporal sequence of antagonist injections (first BMI and then addition of CNQX or vice versa) made no significant difference on the postinjection RF. The statistical difference between the four groups was significant; $p<0.001 . b$, Effects on DSC. Injections of BMI alone (open triangles; also see Fig. $3 c$ ), CNQX alone (open squares: also see Fig. 5 c), and BMI during ongoing (NQX injection (closed diamonds) are shown. Reversing the temporal sequence of antagonist applications, i.e., adding CNQX during ongoing BMI injection, eliminated the initial BMI effect (Fig. $3 c$ ) and resulted in similar blockage of DSC as shown here for CNQX and BMI (BMI + CNQX). For CNQX alone, $n=124$ calls ( 4 cycles); for CNQX and BMI, $n=$ 170 calls ( 4 cycles). c, Average compensation depth plotted relative to the bat's average median RF for CNQX alone (median, $938 \mathrm{~Hz}$ below original RF), BMI alone (median, $620 \mathrm{~Hz}$ above original RF), and BMI during ongoing CNQX injection (median, $4 \mathrm{~Hz}$ above original RF). The differences between all groups were statistically significant; $p<0.001$.
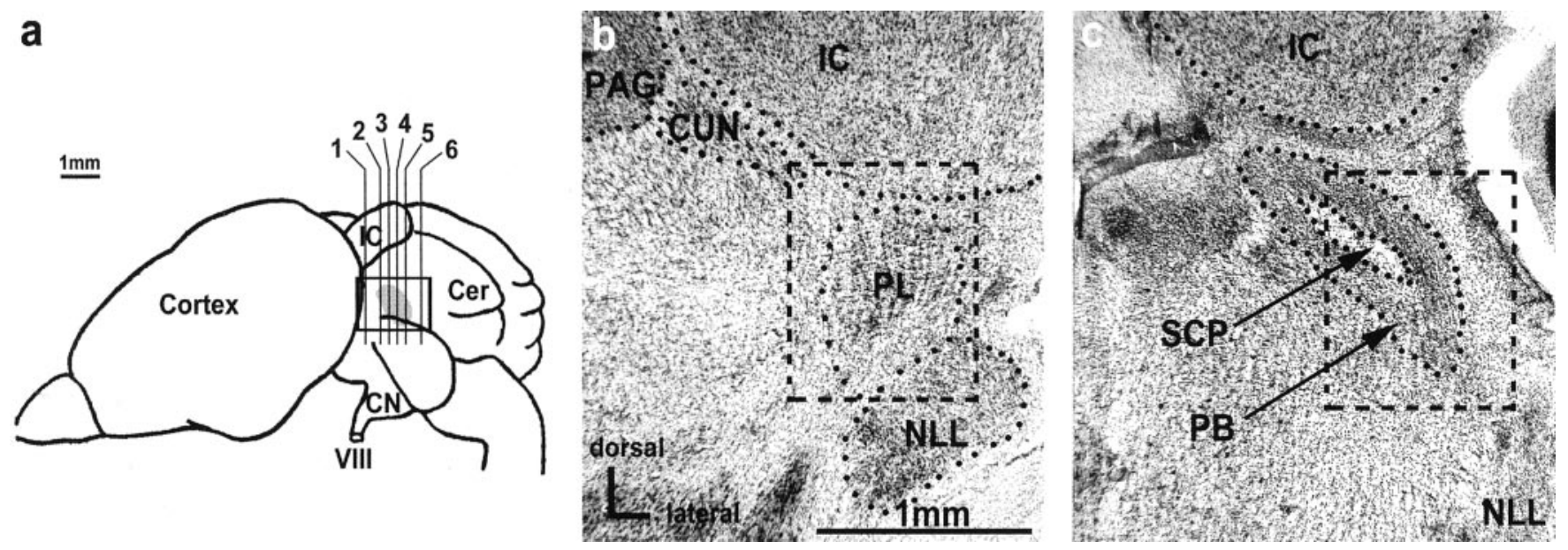

Figure 7. Reconstruction of the location of PB ( gray) in a side view of the horseshoe bat brain ( $a$ ) and histograms of frontal sections through the paralemniscal tegmentum ( $b$ ) and PB ( $c$ ). Numbers in $a$ indicate the positions of sections shown in Figure $8 a_{1}-a_{6}$. Histograms shown in $b$ and c correspond to sections 1 and 3 , respectively. The box in $a$ represents the total area covered by the BMI mapping study shown in Figure 8. The box in $b$ corresponds approximately to the area covered in Figure $8 a_{1}$. The box in c depicts the relative position of PB within the area covered in Figure $8 a_{3}$ but being similar also in Figure $8, a_{2}, a_{4}$, and $a_{5}$. VIII, Auditory nerve; Cer, cerebellum; CN, cochlear nucleus; CUN, cuneiform nucleus; IC, inferior colliculus; NLL, nuclei of the lateral lemniscus; PAG, periaqueductal gray; $P L$, paralemniscal tegmentum; $S C P$, superior cerebellar peduncle.

tions in a total of three bats). BMI injections also caused calls to be produced at rather irregular intervals. Histological verification of the site of small electrolytic lesions set at the conclusion of the experiments and reconstruction of the stereotaxic coordinates indicate that this area is located within the trigeminal nucleus, probably corresponding to its principal sensory nucleus. A few examples of such altered calls are given in Figure 9. In general, BMI caused calls to be produced in distinct pairs of two, normally consisting of a longer first and a shorter second call (Fig. 9a,b). The time interval between calls belonging to one pair was always clearly significantly shorter (always $<10 \mathrm{msec}$ ) than between subsequent doublets $(>20 \mathrm{msec})$. Within one doublet, the first call usually still resembled the typical spectral composition of a horseshoe bat call (Fig. 4), although the initial upward frequency modulation often was prolonged and the spectrum was significantly shifted below RF. In one instance, calls were even emitted at audible frequencies at $\sim 7 \mathrm{kHz}$. (Note that, in contrast, BMI injections into $\mathrm{PB}$ caused call frequencies to rise) (Fig. 3.) The second call consisted of a downward frequency modulation usually covering an up to $15 \mathrm{kHz}$ broader range than normally (compare Figs. $4,9 a, c$ ). It was often preceded by a brief upward sweep (Fig. $9 a, c$ ), which in some cases even bridged the gap between the two calls of a doublet (Fig. 9c). These changes in call structure were quite variable even within a single call sequence lasting for tens of seconds. When playing back frequency-shifted echo mimics, we did not observe any DSC response. Even when the bat was swung on a pendulum, it failed to compensate for frequencyshifted echoes. In addition to the effects on call structure, BMI 


\section{a Frontal:}
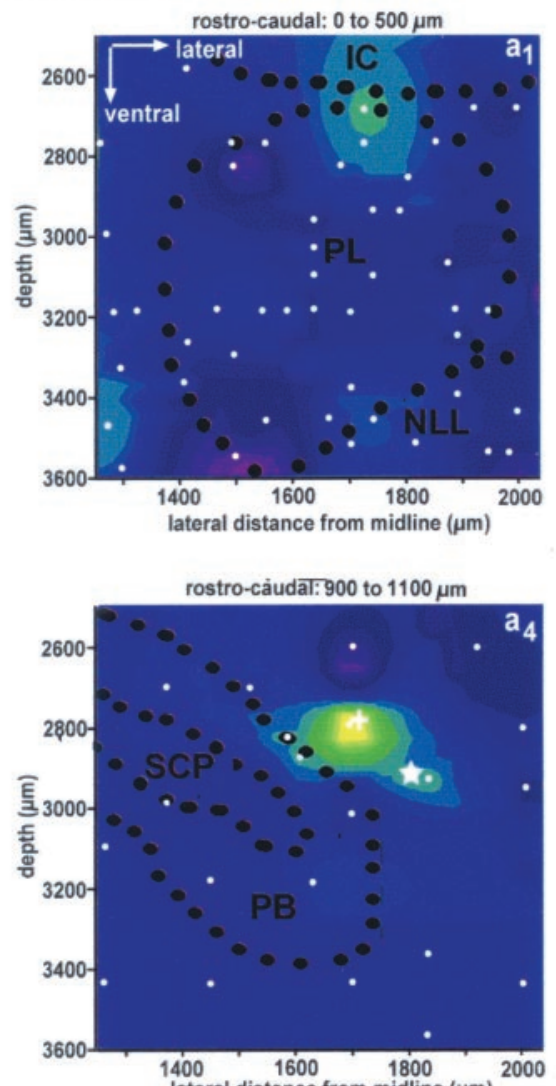

lateral distance from midline $(\mu \mathrm{m})$
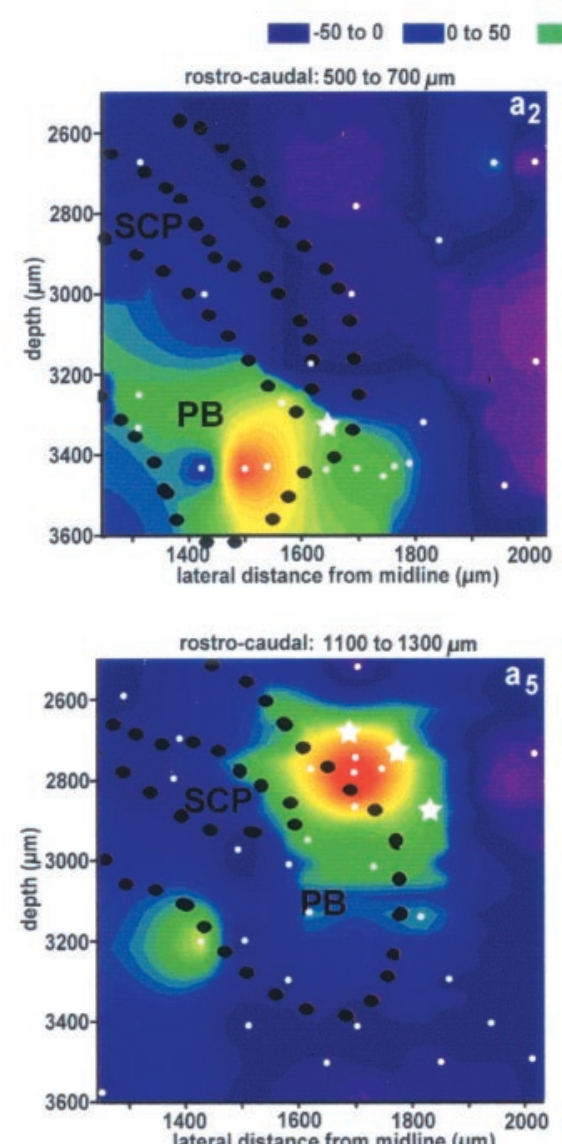

lateral distance from midline $(\mu \mathrm{m})$
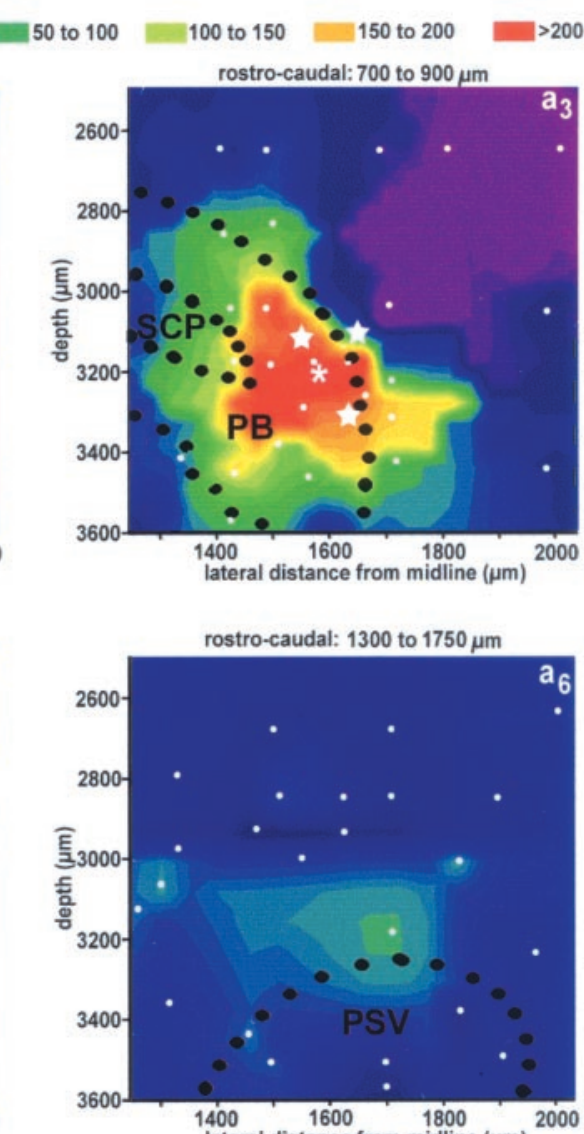

\section{b Horizontal:}
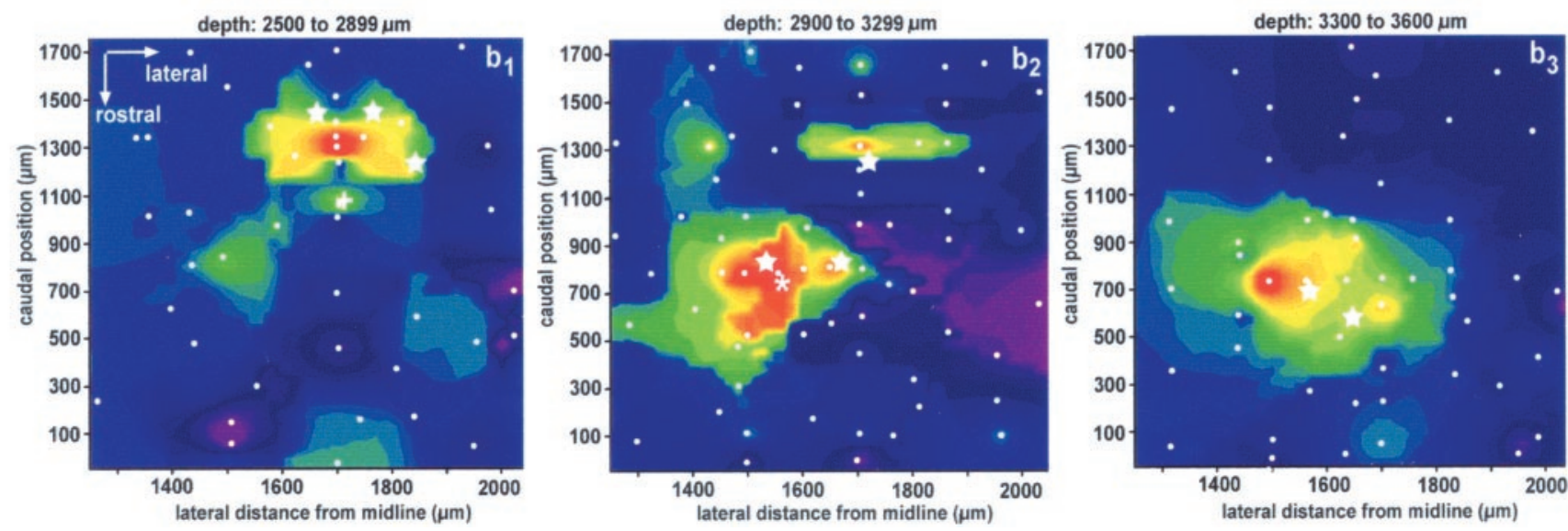

Figure 8. Mapping of call frequency changes during BMI iontophoresis into the midbrain and hindbrain tegmentum as indicated in Figure $7 a$. The scale for the amount of call frequency shifts relative to the preinjection RF is indicated at the top. The weighted average of neighboring data points was computed using the inverse distance method (sampling proportion, 0.3 ; single exponent; SigmaPlot). White dots represent the reconstructed locations of 172 injection sites in two bats for whom closely overlapping regions of the midbrain were investigated in similar detail. The asterisks in $a_{3}$ and $b_{2}$ correspond to the injection site verified in Figure $2 a$; the crosses in $a_{4}$ and $b_{7}$ correspond to the site verified in Figure $2 b$. $a$, Consecutive frontal sections from rostral $\left(a_{7}\right)$ to caudal $\left(a_{6}\right)$ as indicated in Figure 7a. The rostrocaudal boundaries of each section are given relative to the position of the most anterior penetration (Fig. 7a, plane 1). The depth is relative to the surface of the brain. Relevant anatomical landmarks are outlined in black. Note that sections $a_{7}$ and $a_{6}$ cover 500 and $450 \mu \mathrm{m}$, respectively, whereas $a_{2}-a_{5}$ each cover only $200 \mu \mathrm{m} . b$, Horizontal sections from dorsal $\left(b_{1}\right)$ to ventral $\left(b_{3}\right)$. The caudal position was normalized to the position of the most anterior penetration. Same convention as in $a$. White stars localize the most effective stimulation sites found for eight other bats in which a detailed anatomical reconstruction of injections sites was performed. IC, Inferior colliculus; NLL, nuclei of the lateral lemniscus; PL, paralemniscal tegmentum; PSV, principle sensory nucleus of the trigeminal; SCP, superior cerebellar peduncle.

also caused a marked twitching of the horseshoe-shaped nose leaf, mostly contralaterally to the injection side. We did not observe any systematic effects on ear movements. All effects occurred with a latency of up to $20 \mathrm{sec}$ after the onset of drug iontophoresis and were reversible, with normal calling behavior resuming $\sim 20 \mathrm{~min}$ after the end of the injection. Injections of MUS, on the other hand, did not yield any obvious deleterious effects on calling behavior or nose leaf movements, and, except for frequency, the structure of the calls produced were indistinguishable from normal calls. 
a
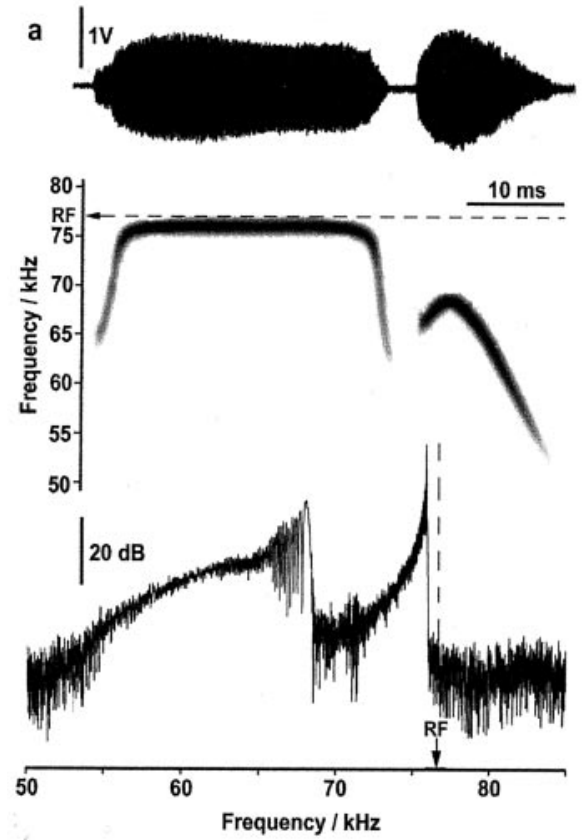

b
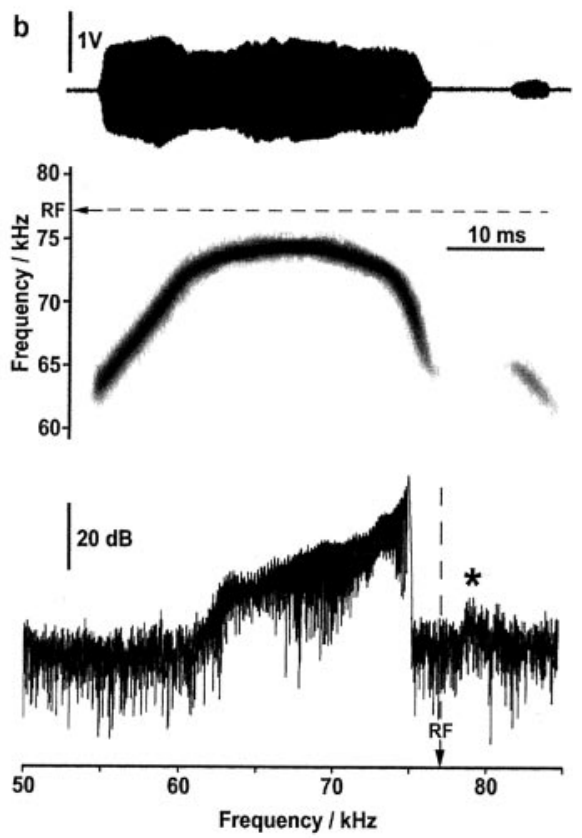

c
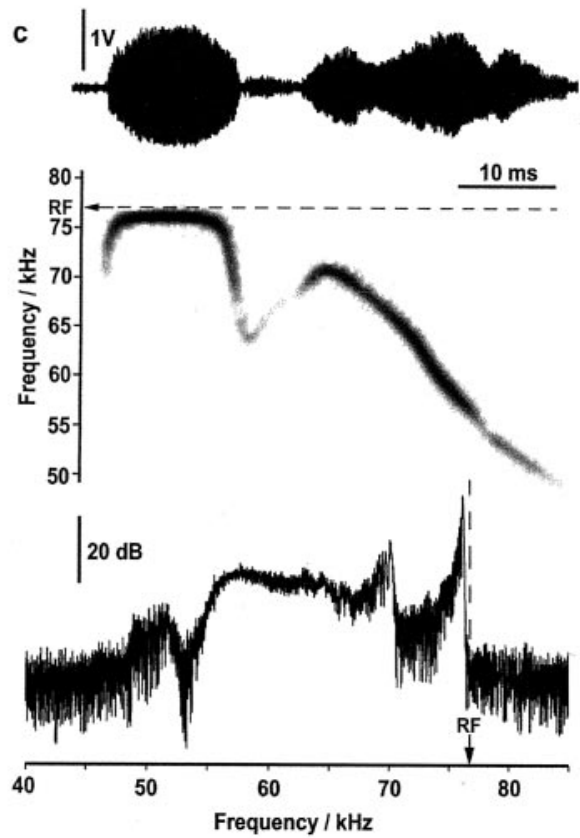

Figure 9. Preliminary results demonstrating the effects of BMI iontophoresis into the trigeminal nucleus, probably its principal sensory nucleus, on the spectral composition of three pairs of echolocation calls $(a-c)$. This area was located $\sim 500 \mu \mathrm{m}$ caudal and $200 \mu \mathrm{m}$ ventral to the PB injection site depicted in Figure $2 a$. Same convention as in Figure 4 . Note the appearance of double calls and dramatic changes in spectral contour. See Figure 4 for example of a typical call under control conditions. See Resultsfor details.

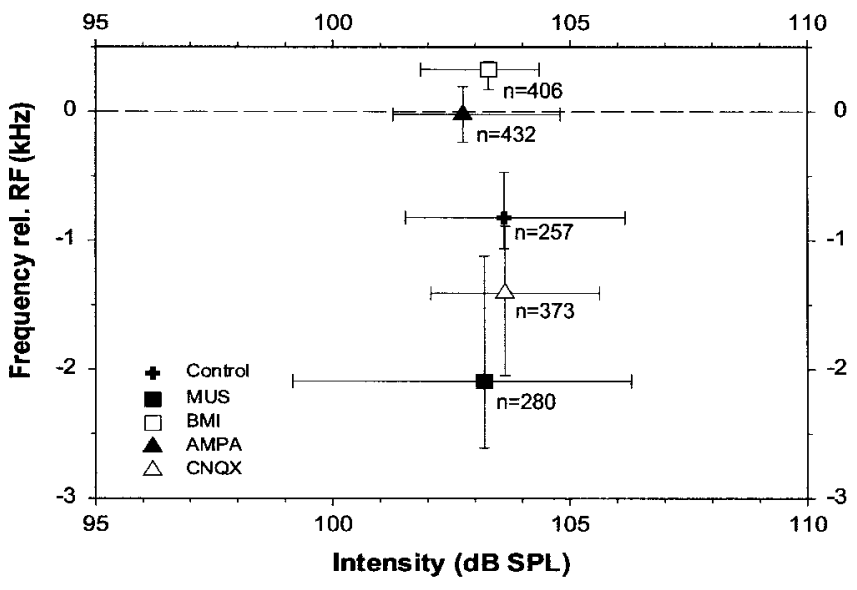

Figure 10. Effects of GABAergic (MUS and BMI) and glutamatergic (AMPA and CNQX) drugs on call frequency ( $y$-axis) and call intensity ( $x$-axis) produced during DSC. Same data as in Figures $3, b(M U S)$ and $c(B M I)$, and $5, b$ (AMPA, Control), and $c(C N Q X)$. Whereas the differences in the call frequencies between all groups were statistically significant $(p<0.001$; also see Figs. $3 d, 5 d$ ), the differences in call intensities were not. The choice of the control group (i.e., controls in Figs. $3 b, c, 5 b, c)$ did not affect this result.

\section{Effects of GABAergic and glutamatergic drugs on call intensity}

We also tested whether the call frequency changes that we observed in response to drug injections into PB were correlated with changes in call intensity. For this purpose, we plotted the same call frequency data obtained during the experiments depicted in Figures $3, b$ and $c$, and $5, b$ and $c$, against the absolute call intensity (Fig. 10). Whereas the median frequencies for the different drug injections were significantly different from one another (also see Figs. $3 d, 5 d$ ), the associated call intensities were not. The analysis of additional call parameters, such as duration and repetition rate, is currently under way, and the results will be presented elsewhere.

\section{Discussion}

The results presented here provide novel evidence for a neural substrate and potential mechanisms for auditory feedback control of call frequencies in echolocating horseshoe bats and potentially mammals in general. We found that within $\mathrm{PB}$, creating a large inhibitory effect by injecting MUS or inhibiting excitation with CNQX both lowered call frequencies emitted at rest and during DSC. Conversely, generating a large excitation with AMPA or blocking inhibition with BMI resulted in increasing call frequencies. This suggests that a base level of activity in PB was necessary to produce normal RF. When the activity in this region was, for instance, enhanced, either by blocking inhibition with BMI or generating excitation with AMPA, RF increased above its normal values, and during DSC, the response to increased playback frequencies was dramatically suppressed or even completely abolished. When the activity was reduced, by either generating inhibition with MUS or blocking excitation with CNQX, RF was lowered, and during DSC, average call frequencies remained below normal values throughout the compensation cycle.

$\mathrm{PB}$ is a highly heterogeneous brainstem structure consisting, in the rat, of at least six subnuclei (Swanson, 1992; Paxinos and Watson, 1998). Its anatomical connectivity and neurophysiological properties are equally intricate. $\mathrm{PB}$ has been shown in various mammals to provide direct afferents to the nucleus retroambiguus (Gerrits and Holstege, 1996; Holstege et al., 1997; Vanderhorst et al., 2000), which in turn projects to the motor nucleus of vocalization control, the nucleus ambiguus (Holstege, 1989; Zhang et al., 1992, 1995). A major source of afferent input to PB appears to be the solitary tract nucleus (Herbert et al., 1990; Ezure et al., 1998). Neurophysiological investigations of PB in cat and monkey revealed that its neuronal activity correlated with numerous aspects related to breathing and vocalization behavior (Larson and Kistler, 1986; Kirzinger and Jürgens, 1991; Larson, 1991; Farley et al., 1992; Larson et al., 1994). Glutamate and electrical microstimulation of the ventrolateral PB in rat (i.e., the 
Koelliker-Fuse subnucleus) changed the respiratory rhythm (Chamberlin and Saper, 1994). These studies therefore suggest that the role of $\mathrm{PB}$ in the control of vocalization might be quite complex, involving a convergence of various sensory and premotor commands, such as from respiratory, vocalization-related, acoustic, and somatosensory brain areas (Farley et al., 1992). In general, however, PB has been considered to play a merely indirect role in vocalization control by participating mainly in the coordination of vocal onset with respiration (Jürgens, 2002). Yet in all mammals studied, including humans, the final motor control of call frequency is ultimately independent of respiratory control or even other vocalization-related parameters, such as call duration and repetition rate (Schuller and Suga, 1976; Rübsamen and Schuller, 1981; Schuller and Rübsamen, 1981; Schweizer et al., 1981; Yajima and Hayashi, 1983; Rübsamen and Betz, 1986; Rübsamen and Schweizer, 1986; Larson et al., 1987).

How then do our results tie into the current picture of $\mathrm{PB}$ ? The effects that GABAergic and glutamatergic drug injections into the horseshoe bat's PB had on the control of call frequencies seemed to us to be too specific to merely be a byproduct of respiratory control. Nevertheless, it has been suggested that in monkey and humans, producing higher call frequencies requires larger expiratory forces than calling at lower frequencies (Titze, 1989; Hausler, 2000). Larger forces of expiration result in a larger subglottic pressure, which indeed has been found to correlate with both higher call intensity and frequency in the larynx of various mammals (Fattu and Suthers, 1981; Titze, 1989; Lancaster et al., 1995; Hausler, 2000). However, our drug injections into PB did not yield any significant changes in call intensity, which is the vocalization parameter most strongly affected by respiration. In addition, PB has generally been implicated in participating in controlling the rate of respiration and, to a lesser extent, the expiratory (or inspiratory) force (Chamberlin and Saper, 1994; Jürgens, 2002). We concluded therefore that the call frequency changes that we observed during our drug injections into PB directly affected the neural circuitry involved in frequency control and were not effects of indirect respiratory control. The extremely heterogeneous organization of $\mathrm{PB}$, the complex spectral composition of the vocalizations uttered by most experimental animals used in previous studies on PB function, and the focus of most studies on topics other than vocalization control could have easily concealed the effects described here.

The PB therefore appears to be part of a complex midbrain network that controls various vocal motor patterns in mammals in general. This network potentially includes several midbrain structures, such as the superior colliculus, the periaqueductal gray, and areas laterally and ventrally adjacent to the periaqueductal gray (Suga et al., 1973; Jürgens and Pratt, 1979; Larson and Kistler, 1986; Rübsamen and Betz, 1986; Thoms and Jürgens, 1987; Schuller and Radtke-Schuller, 1990; Kirzinger and Jürgens, 1991; Larson, 1991; Jürgens and Lu, 1993; Gerrits and Holstege, 1996; Schuller et al., 1997; Jürgens, 1998, 2000, 2002; Behrend and Schuller, 2000). This midbrain network can function independently from higher-order structures of vocalization control, such as the cingulate cortex (Movchan and Burikova, 1982; Movchan, 1984; Gaioni et al., 1990; Riquimaroux et al., 1992), and lesions at the level of the midbrain dramatically affect sound production in various mammals (Movchan, 1980; Movchan and Burikova, 1982; Kirzinger and Jürgens, 1985; Schuller, 1986; Konstantinov et al., 1988; Jürgens, 1998, 2002). Previously, oftenneglected studies in horseshoe bats suggested that after bilateral ablation of the deep layers of the inferior colliculus and ventrally adjacent portions of the tegmentum including $\mathrm{PB}$, call frequen- cies emitted at rest and during DSC became less stable and eliminated DSC behavior or even "inverted" the response; instead of decreasing its vocalization frequency in response to increasing echo frequencies, the bat's vocalization frequency increased on average $1 \mathrm{kHz}$ above RF (Movchan, 1984; Konstantinov et al., 1988). Similarly, DSC behavior in horseshoe bats could be reversibly suppressed by electrically stimulating an area ventral to the inferior colliculus (Schuller, 1986). In none of these previous studies, however, was the localization of the lesion or stimulation sites sufficiently accurate to decide decisively which brain structures were essential for the control of vocalization behavior. More recently, it has been shown that the DSC response could be diminished by lesions of the nucleus of the central acoustic tract (Kobler et al., 1987; Behrend and Schuller, 2000); previously described as the anterolateral periolivary nucleus). In addition to relaying auditory information relevant for DSC to areas outside the classical auditory pathway, such as the pretectal area and superior colliculus, the central acoustic tract probably represents a sensory pathway that affects other behaviors as well (Behrend and Schuller, 2000). Finally, our own previous neurophysiological and anatomical investigations suggested that the paralemniscal tegmentum could be involved in auditory feedback control of call frequencies (Metzner, 1989, 1993, 1996), yet electrolytic and pharmacological lesions did not support direct involvement (Schuller et al., 1997; Pillat and Schuller, 1998). Similarly, we observed no changes in DSC or RF when injecting GABAergic and glutamatergic drugs into the paralemniscal tegmentum. Therefore, although there are reliable indications that the tegmental region is functionally associated with some aspects of vocalization and echolocation (Schuller et al., 1997; Pillat and Schuller, 1998), its normal functioning does not appear to be critical to DSC or the control over RF.

Because both the auditory system (Pollak and Casseday, 1989; Popper and Fay, 1992, 1995; Webster et al., 1992) and the vocalization system (Suga et al., 1973; Rübsamen and Schuller, 1981; Schuller and Rübsamen, 1981; Schweizer et al., 1981; Yajima and Hayashi, 1983; Rübsamen and Betz, 1986; Rübsamen and Schweizer, 1986; Gooler and O’Neill, 1987; Yajima and Larson, 1993; Jürgens, 1998, 2002) are separately built on common structural and functional elements in all mammals studied so far, DSC behavior is likely to share basic aspects with audio-vocal feedback control of vocal pitch in other mammals (Janik and Slater, 1997), including the involuntary response to "pitch-shifted feedback" in humans (Elman, 1981; Burnett et al., 1998; Houde and Jordan, 1998; Donath et al., 2002).

Whereas auditory feedback does not seem to affect vocalizations in various adult nonhuman primates and in adult cats (Talmage-Riggs et al., 1972; Winter et al., 1973; Romand and Ehret, 1984; Shipley et al., 1988; Janik and Slater, 1997; Jürgens, 1998), it is essential in bats (Griffin, 1986; Boughman, 1998). Thus, DSC in horseshoe bats can provide a valuable animal model for dissecting the neural basis for auditory feedback control of mammalian vocalization and possibly vertebrates in general (Bass et al., 1997; Janik and Slater, 1997; Wild, 1997a,b; Burnett et al., 1998; Houde and Jordan, 1998; Jürgens, 1998; Donath et al., 2002). Specifically, DSC behavior in horseshoe bats has provided us with a broader appreciation for the functional significance of a prominent structure in the mammalian brainstem, the $\mathrm{PB}$, which appears to be essential for something as crucial as the control of call frequencies by altered auditory feedback in echolocating horseshoe bats. 


\section{References}

Bass AH, Bodnar DA, McKibben JR (1997) From neurons to behavior: vocal-acoustic communication in teleost fish. Biol Bull 192:158-160.

Behrend O, Schuller G (2000) The central acoustic tract and audio-vocal coupling in the horseshoe bat, Rhinolophus rouxi. Eur J Neurosci 12:4268-4280.

Boughman JW (1998) Vocal learning by greater spear-nosed bats. Proc R Soc Lond B Biol Sci 265:227-233.

Burnett TA, Freedland MB, Larson CR, Hain TC (1998) Voice F0 responses to manipulations in pitch feedback. J Acoust Soc Am 103:3153-3161.

Chamberlin NL, Saper CB (1994) Topographic organization of respiratory responses to glutamate microstimulation of the parabrachial nucleus in the rat. J Neurosci 14:6500-6510.

Donath TM, Natke U, Kalveram KT (2002) Effects of frequency-shifted auditory feedback on voice F0 contours in syllables. J Acoust Soc Am 111:357-366.

Elman JL (1981) Effects of frequency-shifted feedback on the pitch of vocal productions. J Acoust Soc Am 70:45-50.

Esser KH (1994) Audio-vocal learning in a non-human mammal: the lesser spear-nosed bat, Phyllostomus discolor. NeuroReport 5:1718-1720.

Ezure K, Tanaka I, Miyazaki M (1998) Pontine projections of pulmonary slowly adapting receptor relay neurons in the cat. NeuroReport 9:411-414.

Farley GR, Barlow SM, Netsell R (1992) Factors influencing neural activity in parabrachial regions during cat vocalizations. Exp Brain Res 89:341-351

Fattu JM, Suthers RA (1981) Subglottic pressure and the control of phonation by the echolocating bat, Eptesicus. J Comp Physiol [A] 143:465-475.

Gaioni SJ, Riquimaroux H, Suga N (1990) Biosonar behavior of mustached bats swung on a pendulum prior to cortical ablation. J Neurophysiol 64:1801-1817.

Gerrits PO, Holstege G (1996) Pontine and medullary projections to the nucleus retroambiguus: a wheat germ agglutinin-horseradish peroxidase and autoradiographic tracing study in the cat. J Comp Neurol 373:173-185.

Gooler DM, O’Neill WE (1987) Topographic representation of vocal frequency demonstrated by microstimulation of anterior cingulate cortex in the echolocating bat, Pteronotus parnelli parnelli. J Comp Physiol [A] 161:283-294.

Griffin DR (1986) Listening in the dark: the acoustic orientation of bats and men. Ithaca, NY: Comstock.

Grinnell AD (1989) Sensory-motor control: listening to the voice within. Nature 341:488-489.

Hausler U (2000) Vocalization-correlated respiratory movements in the squirrel monkey. J Acoust Soc Am 108:1443-1450.

Herbert H, Moga MM, Saper CB (1990) Connections of the parabrachial nucleus with the nucleus of the solitary tract and the medullary reticular formation in the rat. J Comp Neurol 293:540-580.

Holstege G (1989) Anatomical study of the final common pathway for vocalization in the cat. J Comp Neurol 284:242-252.

Holstege G, Kerstens L, Moes MC, Vanderhorst VG (1997) Evidence for a periaqueductal gray-nucleus retroambiguus-spinal cord pathway in the rat. Neuroscience 80:587-598.

Houde JF, Jordan MI (1998) Sensorimotor adaptation in speech production. Science 279:1213-1216.

Janik VM, Slater PJB (1997) Vocal learning in mammals. In: Advances in the study of behavior, Vol 26 (Slater PJB, Rosenblatt JS, Snowdon CT, Milinski M, eds), pp 59-99. San Diego: Academic.

Jürgens U (1998) Neuronal control of mammalian vocalization, with special reference to the squirrel monkey. Naturwissenschaften 85:376-388.

Jürgens U (2000) Localization of a pontine vocalization-controlling area. J Acoust Soc Am 108:1393-1396.

Jürgens U (2002) Neural pathways underlying vocal control. Neurosci Biobehav Rev 26:235-258.

Jürgens U, Lu CL (1993) The effects of periaqueductally injected transmitter antagonists on forebrain-elicited vocalization in the squirrel monkey. Eur J Neurosci 5:735-741.

Jürgens U, Pratt R (1979) The cingular vocalization pathway in the squirrel monkey. Exp Brain Res 34:499-510.

Kirzinger A, Jürgens U (1985) The effects of brainstem lesions on vocalization in the squirrel monkey. Brain Res 358:150-162.
Kirzinger A, Jürgens U (1991) Vocalization-correlated single-unit activity in the brain stem of the squirrel monkey. Exp Brain Res 84:545-560.

Kobler JB, Isbey SF, Casseday JH (1987) Auditory pathways to the frontal cortex of the mustache bat, Pteronotus parnellii. Science 236:824-826.

Konstantinov AI, Makarov AK, Movchan EV, Sokolov BV, Goriniskii IA (1988) Sensory system for echolocation in horseshoe bats (in Russian). Moscow: Russian Academy of Sciences.

Lancaster WC, Henson Jr OW, Keating AW (1995) Respiratory muscle activity in relation to vocalization in flying bats. J Exp Biol 198:175-191.

Larson CR (1991) On the relation of PAG neurons to laryngeal and respiratory muscles during vocalization in the monkey. Brain Res 552:77-86.

Larson CR, Kistler MK (1986) The relationship of periaqueductal gray neurons to vocalization and laryngeal EMG in the behaving monkey. Exp Brain Res 63:596-606.

Larson CR, Kempster GB, Kistler MK (1987) Changes in voice fundamental frequency following discharge of single motor units in cricothyroid and thyroarytenoid muscles. J Speech Hear Res 30:552-558.

Larson CR, Yajima Y, Ko P (1994) Modification in activity of medullary respiratory-related neurons for vocalization and swallowing. J Neurophysiol 71:2294-2304.

McCowan B, Reiss D (1997) Vocal learning in captive bottlenose dolphins: a comparison with humans and nonhuman animals. In: Social influences on vocal development (Snowden CT, Hausberger M, eds), pp 178-207. Cambridge, UK: Cambridge UP.

Metzner W (1989) A possible neuronal basis for Doppler-shift compensation in echo-locating horseshoe bats. Nature 341:529-532.

Metzner W (1993) An audio-vocal interface in echolocating horseshoe bats. J Neurosci 13:1899-1915.

Metzner W (1996) Anatomical basis for audio-vocal integration in echolocating horseshoe bats. J Comp Neurol 368:252-269.

Metzner W, Juranek J (1997) A method to biotinylate and histochemically visualize ibotenic acid for pharmacological inactivation studies. J Neurosci Methods 76:143-150.

Metzner W, Zhang SY, Smotherman MS (2002) Doppler-shift compensation behavior in horseshoe bats revisited: auditory feedback controls both a decrease and an increase in call frequency. J Exp Biol 205:1607-1616.

Movchan EV (1980) Effects of destruction of the inferior colliculus on function of the echolocation system in horseshoe bats. Neirofiziologiia 12:375-381.

Movchan EV (1984) Participation of the auditory centers of Rhinolophus ferrumequinum in echolocational tracking of a moving target. Neirofiziologiia 16:737-745.

Movchan EV, Burikova NV (1982) Effect of auditory cortical ablation on functioning of the Doppler echolocation system in horseshoe bats. Neirofiziologiia 14:43-50.

Neuweiler G, Metzner W, Heilmann U, Rubsamen R, Eckrich M, Costa HH (1987) Foraging behaviour and echolocation in the rufous horseshoe bats, Rhinolophus rouxi, of Sri Lanka. Behav Ecol Sociobiol 20:53-67.

Paxinos G, Watson C (1998) The rat brain in stereotaxic coordinates. San Diego: Academic.

Pillat J, Schuller G (1998) Audiovocal behavior of Doppler-shift compensation in the horseshoe bat survives bilateral lesion of the paralemniscal tegmental area. Exp Brain Res 119:17-26.

Pollak GD, Casseday JH (1989) The neural basis of echolocation in bats. Berlin: Springer.

Popper AN, Fay RR, eds (1992) The mammalian auditory pathway: neurophysiology. New York: Springer.

Popper AN, Fay RR, eds (1995) Hearing by bats. New York: Springer.

Riquimaroux H, Gaioni SJ, Suga N (1992) Inactivation of the DSCF area of the auditory cortex with muscimol disrupts frequency discrimination in the mustached bat. J Neurophysiol 68:1613-1623.

Romand R, Ehret G (1984) Development of sound production in normal, isolated, and deafened kittens during the first postnatal months. Dev Psychobiol 17:629-649.

Rübsamen R, Betz M (1986) Control of echolocation pulses by neurons of the nucleus ambiguus in the rufous horseshoe bat, Rhinolophus rouxi. I. Single unit recordings in the ventral motor nucleus of the laryngeal nerves in spontaneously vocalizing bats. J Comp Physiol [A] 159:675-687.

Rübsamen R, Schäfer M (1990) Audiovocal interactions during development? Vocalisation in deafened young horseshoe bats vs. audition in vocalisation-impaired bats. J Comp Physiol [A] 167:771-784.

Rübsamen R, Schuller G (1981) Laryngeal nerve activity during pulse emis- 
sion in the CF-FM bat, Rhinolophus ferrumequinum. II. The recurrent laryngeal nerve. J Comp Physiol [A] 143:323-327.

Rübsamen R, Schweizer H (1986) Control of echolocation pulses by neurons of the nucleus ambiguus in the rufous horseshoe bat, Rhinolophus rouxi. II. Afferent and efferent connections of the motor nucleus of the laryngeal nerves. J Comp Physiol [A] 159:689-699.

Schnitzler HU (1968) Die Ultraschallortungslaute der HufeisennasenFledermäuse (Chiroptera, Rhinolophidae) in verschiedenen Orientierungssituationen. Z Vergl Physiol 57:376-408.

Schuller G (1986) Influence of echolocation pulse rate on Doppler-shift compensation control system in the greater horseshoe bat. J Comp Physiol [A] 158:239-246.

Schuller G, Pollak GD (1979) Disproportionate frequency representation in the inferior colliculus of Doppler-compensating greater horseshoe bats, Rhinolophus ferrumequinum. J Comp Physiol [A] 132:47-54.

Schuller G, Radtke-Schuller S (1990) Neural control of vocalization in bats: mapping of brainstem areas with electrical microstimulation eliciting species-specific echolocation calls in the rufous horseshoe bat. Exp Brain Res 79:192-206.

Schuller G, Rübsamen R (1981) Laryngeal nerve activity during pulse emission in the CF-FM bat, Rhinolophus ferrumequinum. I. Superior laryngeal nerve. J Comp Physiol [A] 143:317-321.

Schuller G, Suga N (1976) Laryngeal mechanism for the emission of CF-FM sounds in the Doppler-shift compensating greater horseshoe bat, Rhinolophus ferrumequinum. J Comp Physiol [A] 107:253-262.

Schuller G, Beuter K, Schnitzler HU (1974) Response to frequency-shifted artificial echoes in the bat, Rhinolophus ferrumequinum. J Comp Physiol [A] 89:275-286.

Schuller G, Beuter K, Rübsamen R (1975) Dynamic properties of the compensation system for Doppler-shifts in the bat, Rhinolophus ferrumequinum. J Comp Physiol [A] 97:113-125.

Schuller G, Radtke-Schuller S, Betz M (1986) A stereotaxic method for small animals using experimentally determined reference profiles. J Neurosci Methods 18:339-350.

Schuller G, Fischer S, Schweizer H (1997) Significance of the paralemniscal tegmental area for audio-motor control in the moustached bat, Pteronotus p. parnellii: the afferent off efferent connections of the paralemniscal area. Eur J Neurosci 9:342-355.

Schweizer H, Rübsamen R, Ruhle C (1981) Localization of brain stem motoneurones innervating the laryngeal muscles in the rufous horseshoe bat, Rhinolophus rouxi. Brain Res 230:41-50.

Shipley C, Buchwald JS, Carterette EC (1988) The role of auditory feedback in the vocalizations of cats. Exp Brain Res 69:431-438.
Simmons JA (1974) Response of the Doppler echolocation system in the bat, Rhinolophus ferrumequinum. J Acoust Soc Am 56:672-682.

Smotherman M, Metzner W (2003) Effects of echo intensity on Doppler-shift compensation behavior in horseshoe bats. J Neurophysiol 89:814-821.

Suga N, Schlegel P, Shimozawa T, Simmons J (1973) Orientation sounds evoked from echolocating bats by electrical stimulation of the brain. J Acoust Soc Am 54:793-797.

Swanson LW (1992) Brain maps: structure of the rat brain. Amsterdam: Elsevier.

Talmage-Riggs G, Winter P, Ploog D, Mayer W (1972) Effect of deafening on the vocal behavior of the squirrel monkey (Saimiri sciureus). Folia Primatol 17:404-420.

Thoms G, Jürgens U (1987) Common input of the cranial motor nuclei involved in phonation in squirrel monkey. Exp Neurol 95:85-99.

Tian B, Schnitzler HU (1997) Echolocation signals of the greater horseshoe bat (Rhinolophus ferrumequinum) in transfer flight and during landing. I Acoust Soc Am 101:2347-2364.

Titze IR (1989) On the relation between subglottal pressure and fundamental frequency in phonation. J Acoust Soc Am 85:901-906.

Vanderhorst VG, Terasawa E, Ralston III HJ, Holstege G (2000) Monosynaptic projections from the nucleus retroambiguus to motoneurons supplying the abdominal wall, axial, hindlimb, and pelvic floor muscles in the female rhesus monkey. J Comp Neurol 424:233-250.

Webster DB, Popper AN, Fay RR (1992) The mammalian auditory pathway: neuroanatomy. New York: Springer.

Wild JM (1997a) Functional anatomy of neural pathways contributing to the control of song production in birds. Eur J Morphol 35:303-325.

Wild JM (1997b) Neural pathways for the control of birdsong production. J Neurobiol 33:653-670.

Winter P, Handley P, Ploog D, Schott D (1973) Ontogeny of squirrel monkey calls under normal conditions and under acoustic isolation. Behaviour 47:230-239.

Yajima Y, Hayashi Y (1983) Ambiguus motoneurons discharging synchronously with ultrasonic vocalization in rats. Exp Brain Res 50:359-366.

Yajima Y, Larson CR (1993) Multifunctional properties of ambiguus neurons identified electrophysiologically during vocalization in the awake monkey. J Neurophysiol 70:529-540.

Zhang SP, Davis PJ, Carrive P, Bandler R (1992) Vocalization and marked pressor effect evoked from the region of the nucleus retroambigualis in the caudal ventrolateral medulla of the cat. Neurosci Lett 140:103-107.

Zhang SP, Bandler R, Davis PJ (1995) Brain stem integration of vocalization: role of the nucleus retroambigualis. J Neurophysiol 74:2500-2512. 